\title{
EXTREMAL BEHAVIOUR OF HITTING A CONE BY CORRELATED BROWNIAN MOTION WITH DRIFT
}

\author{
KRZYSZTOF DȨBICKI, ENKELEJD HASHORVA, LANPENG JI, AND TOMASZ ROLSKI
}

\begin{abstract}
This paper derives an exact asymptotic expression for

$$
\mathbb{P}_{\mathbf{x}_{u}}\left\{\exists_{t \geq 0} \mathbf{X}(t)-\boldsymbol{\mu} t \in \mathcal{U}\right\}, \text { as } u \rightarrow \infty
$$

where $\mathbf{X}(t)=\left(X_{1}(t), \ldots, X_{d}(t)\right)^{\top}, t \geq 0$ is a correlated $d$-dimensional Brownian motion starting at the point $\mathbf{x}_{u}=-\boldsymbol{\alpha} u$ with $\boldsymbol{\alpha} \in \mathbb{R}^{d}, \boldsymbol{\mu} \in \mathbb{R}^{d}$ and $\mathcal{U}=\prod_{i=1}^{d}[0, \infty)$. The derived asymptotics depends on the solution of an underlying multidimensional quadratic optimization problem with constraints, which leads in some cases to dimension-reduction of the considered problem. Complementary, we study asymptotic distribution of the conditional first passage time to $\mathcal{U}$, which depends on the dimension-reduction phenomena.
\end{abstract}

Key Words: multidimensional Brownian motion; extremes; exact asymptotics; first passage time; large deviations; quadratic programming problem; multidimensional Pickands constants.

AMS Classification: Primary 60G15; secondary 60G70

\section{INTRODUCTION}

Consider $\boldsymbol{X}(t)-\boldsymbol{\mu} t, t \geq 0$, a correlated $d$-dimensional Brownian motion with drift, where $\boldsymbol{X}(t)=A \boldsymbol{B}(t), A \in \mathbb{R}^{d \times d}$ is a non-singular matrix, $\boldsymbol{B}(t)=\left(B_{1}(t), \ldots, B_{d}(t)\right)^{\top}, t \geq 0$ is a standard $d$-dimensional Brownian motion with independent coordinates and $\boldsymbol{\mu}=\left(\mu_{1}, \ldots, \mu_{d}\right)^{\top} \in \mathbb{R}^{d}$.

The probability

$$
\mathbb{P}_{\boldsymbol{x}}\left\{\exists_{t \geq 0} \boldsymbol{X}(t)-\boldsymbol{\mu} t \in \mathcal{U}\right\}
$$

that starting at the point $\boldsymbol{x} \in \mathbb{R}^{d}$, the process $\boldsymbol{X}(t)-\boldsymbol{\mu} t$ enters the set $\mathcal{U} \subset \mathbb{R}^{d}$ in a finite time, is of interest both for theory-oriented studies and for applied-mathematics problems as, e.g., heat and mass diffusion, photon absorption or chemotaxis. Due to the complexity of (1), still only some fragmentary results focusing on the special case of mutually independent coordinates (i.e., for $A$ being the identity matrix) or on particular structures of $\mathcal{U}$ are available. We refer to, e.g., [1] for the asymptotic analysis, as $r:=\|\boldsymbol{x}\| \rightarrow \infty$, of (1) for $A$ the identity matrix, some compact $\mathcal{U}$, and appropriately chosen drifts, see also $[2,3]$. Somehow related problem for the exit time from a cone for a (noncorrelated) multidimensional Brownian motion with drift was considered in [4] and references therein; see also [5] for the case of $\mathcal{U}$ being a Weyl chamber.

This contribution is concerned with investigation of (1) for the model allowing correlation between the Brownian components. More precisely, we investigate the asymptotics of probability that in infinite-time horizon, the process $\boldsymbol{X}(t)-\boldsymbol{\mu} t, t \geq 0$, starting at point $\boldsymbol{x}_{u}:=\left(-\alpha_{1} u, \ldots,-\alpha_{d} u\right)^{\top}$ with $\alpha_{i} \in \mathbb{R}, 1 \leq i \leq d, u>0$, enters the cone $\mathcal{U}=\prod_{i=1}^{d}[0, \infty)$, that is

$$
P(u):=\mathbb{P}_{\boldsymbol{x}_{u}}\left\{\exists_{t \geq 0} \boldsymbol{X}(t)-\boldsymbol{\mu} t \in \mathcal{U}\right\}, \quad u \rightarrow \infty
$$

Date: November 23, 2017. 
Our results allow for considering other sets in (2), as e.g., polyhedral cones $\left\{\boldsymbol{x} \in \mathbb{R}^{d}: M \boldsymbol{x} \geq \mathbf{0}\right\}$, where $M$ is a $d \times d$ non-singular matrix. Indeed, by a linear transformation of $M$, we can reduce the problem of hitting the polyhedral cone to (2), namely

$$
\mathbb{P}_{\boldsymbol{x}_{u}}\left\{\exists_{t \geq 0} \boldsymbol{X}(t)-\boldsymbol{\mu} t \in\left\{\boldsymbol{x} \in \mathbb{R}^{d}: M \boldsymbol{x} \geq \mathbf{0}\right\}\right\}=\mathbb{P}_{\boldsymbol{x}_{u}}\left\{\exists_{t \geq 0} M \boldsymbol{X}(t)-M \boldsymbol{\mu} t \in \prod_{i=1}^{d}[0, \infty)\right\},
$$

with $\boldsymbol{x}_{u}=M \boldsymbol{x}_{u}$.

Since we are interested in the case that $\lim _{u \rightarrow \infty} P(u)=0$ we shall assume that there exists some $1 \leq i \leq d$ such that

$$
\alpha_{i}>0, \quad \mu_{i}>0 .
$$

Using that

$$
P(u)=\mathbb{P}_{\mathbf{0}}\left\{\exists_{t \geq 0} \bigcap_{i=1}^{d}\left\{X_{i}(t)-\mu_{i} t>\alpha_{i} u\right\}\right\}
$$

this paper contributes also to extreme value problems of vector-valued stochastic processes.

Complementary, we investigate distributional properties of the passage time of $\boldsymbol{X}(t)-\boldsymbol{\mu} t$ to $\mathcal{U}$, for $\left\|\boldsymbol{x}_{u}\right\| \rightarrow \infty$ as $u \rightarrow \infty$, given that the multivariate process has ever entered the upper quadrant. Specifically, for

$$
\tau_{u}=\inf \{t \geq 0: \boldsymbol{X}(t)-\boldsymbol{\mu} t>\boldsymbol{\alpha} u\}
$$

$(\mathbf{X}(0)=\mathbf{0})$ we are interested in the approximate distribution of $\tau_{u} \mid \tau_{u}<\infty$ as $u \rightarrow \infty$.

In the 1-dimensional setup it is well-known that for $\alpha, \mu$ positive

$$
P(u)=\mathbb{P}\left\{\sup _{t \geq 0}\left(B_{1}(t)-\mu t\right)>\alpha u\right\}=e^{-2 \alpha \mu u},
$$

where from this point on we write $\mathbb{P}:=\mathbb{P}_{\mathbf{0}}$. Further, in view of [6] we have that

$$
\lim _{u \rightarrow \infty} \mathbb{P}\left\{\alpha^{-1 / 2} \mu^{3 / 2}\left(\tau_{u}-\alpha u / \mu\right) / \sqrt{u} \leq s \mid \tau_{u}<\infty\right\}=\Phi(s), \quad s \in \mathbb{R}
$$

with $\Phi$ the distribution function of an $\mathcal{N}(0,1)$ random variable. Normal or exponential approximations for 1dimensional Gaussian counterparts of the considered model in this contribution are discussed in [6-8].

In the case $d \geq 2$, both the approximation of $P(u)$ and the approximate distribution of $\tau_{u} \mid \tau_{u}<\infty$ depend on the solution of a related quadratic optimization problem. In particular, in the light of [9][Theorem 1], the logarithmic asymptotics of $(2)$ can be derived and takes the following form (hereafter $\sim$ means asymptotic equivalence as $u \rightarrow \infty)$

$$
-\ln P(u) \quad \sim \quad \frac{\widehat{g}}{2} u, \quad \widehat{g}=\inf _{t \geq 0} g(t),
$$

with

$$
g(t)=\frac{1}{t} \inf _{\boldsymbol{v} \geq \boldsymbol{\alpha}+\boldsymbol{\mu} t} \boldsymbol{v}^{\top} \Sigma^{-1} \boldsymbol{v}, \quad \Sigma=A A^{\top} .
$$

Clearly, (5) is of no use for the approximation of the conditional passage time $\tau_{u} \mid \tau_{u}<\infty$ as $u \rightarrow \infty$.

Our main result presented in Theorem 3.1 shows that

$$
P(u) \sim C_{I} \mathcal{H}_{I} u^{\frac{1-m}{2}} e^{-\frac{\hat{g}}{2} u},
$$


where $C_{I}>0, m \in \mathbb{N}$ are known constants and $\mathcal{H}_{I}$ is a multidimensional counterpart of the celebrated Pickands constant that appears in the extreme value theory of Gaussian random fields; see e.g., [10-17]. In Theorem 3.3 we derive approximation of the conditional passage time.

One of the findings of this paper is that the set of indexes $\{1, \ldots, d\}$ of the vector-process $\boldsymbol{X}$ can be partitioned into three subsets $I, J, K$. The index set $I$ determines $m, \widehat{g}$ and $\mathcal{H}_{I}$ in the asymptotics (7), whereas both $I$ and $K$ determine the constant $C_{I}$. Moreover, the set $J$, whenever non-empty, contains indices that do not play any role in our asymptotic consideration. Interestingly, the limit distribution of the conditional passage time derived in Theorem 3.3 is Gaussian only if $K=\emptyset$.

Our investigation shows that for $d \geq 2$, the problem (2) is surprisingly hard even for the seemingly simple case of independent components, that is with $A$ being the identity matrix. Besides, solving this particular case does not reveal the essential ingredients that determine the asymptotics of $P(u)$ in the general case where $A$ is not the identity matrix.

The strategy of the proof of the main result, given in Theorem 3.1, although in its roots based on the double sum technique developed in 1-dimensional setting for extremes of Gaussian processes and fields (see, e.g. [10-12]), needed new ideas that in several key steps of the argumentation significantly differ from methods used in 1-dimensional case. In particular, one of difficulties is the lack of Slepian-type inequalities that could be applied in our vectorvalued setting. Notice also that the standard techniques utilized for proving the negligibility of the double-sum, as e.g., in [12], do not work in the general $d$-dimensional vector-valued case. Other difficulty lies in analysis of the multidimensional Pickands constants $\mathcal{H}_{I}$. Establishing its finiteness and positivity requires significant efforts. The developed in this paper approach opens some possibilities for its application to asymptotic analysis of some related functionals of vector-valued Gaussian processes.

In this contribution we present a full general picture and a complete solution of the problem at hand by developing new techniques building up on asymptotic theory, convex optimization and probability theory. Additionally, we analyze in details some special cases including the case of independent components, the homogeneous case when $\alpha_{j}=\alpha$ and $\mu_{j}=\mu$ for all $j$ and the case with negatively associated components. Moreover, we discuss several interesting special cases when $d=2$.

We organise the paper as follows. The next section fixes the notation and presents some preliminary findings. The main results with examples are presented in Section 3, with detailed proof relegated to Section 4. Detailed analysis of the related optimization problem and some technical proofs are displayed in Appendix.

\section{Preliminaries}

All vectors here are $d$-dimensional column vectors written in bold letters with $d \geq 2$. For instance $\boldsymbol{\alpha}=\left(\alpha_{1}, \ldots, \alpha_{d}\right)^{\top}$, with $^{\top}$ the transpose sign. Operations with vectors are meant component-wise, so $|\boldsymbol{x}|=\left(\left|x_{1}\right|, \ldots,\left|x_{d}\right|\right)^{\top}$ and $\lambda \boldsymbol{x}=\boldsymbol{x} \lambda=\left(\lambda x_{1}, \ldots, \lambda x_{d}\right)^{\top}$ for any $\lambda \in \mathbb{R}, \boldsymbol{x} \in \mathbb{R}^{d}$. We denote

$$
\mathbf{0}=(0, \ldots, 0)^{\top} \in \mathbb{R}^{d}, \quad \mathbf{1}=(1, \ldots, 1)^{\top} \in \mathbb{R}^{d}
$$

For any non-empty subset $\mathcal{T} \subset \mathbb{R}$, denote the inner set of $\mathcal{T}$ by $\mathcal{T}^{o}$ and its closure set by $\overline{\mathcal{T}}$. If $I \subset\{1, \ldots, d\}$, then for a vector $\boldsymbol{a} \in \mathbb{R}^{d}$ we denote by $\boldsymbol{a}_{I}=\left(a_{i}, i \in I\right)$ a sub-block vector of $\boldsymbol{a}$. Similarly, if further $J \subset\{1, \ldots, d\}$, for a matrix $M=\left(m_{i j}\right)_{i, j \in\{1, \ldots, d\}} \in \mathbb{R}^{d \times d}$ we denote by $M_{I J}=M_{I, J}=\left(m_{i j}\right)_{i \in I, j \in J}$ the sub-block matrix of $M$ 
determined by $I$ and $J$. Further, write $M_{I I}^{-1}=\left(M_{I I}\right)^{-1}$ for the inverse matrix of $M_{I I}$ whenever it exists. The next lemma stated in [18] (see also [19]) is important for several definitions in the sequel.

Lemma 2.1. Let $M \in \mathbb{R}^{d \times d}, d \geq 2$ be a positive definite matrix. If $\boldsymbol{b} \in \mathbb{R}^{d} \backslash(-\infty, 0]^{d}$, then the quadratic programming problem

$$
P_{M}(\boldsymbol{b}) \text { : minimise } \boldsymbol{x}^{\top} M^{-1} \boldsymbol{x} \text { under the linear constraint } \boldsymbol{x} \geq \boldsymbol{b}
$$

has a unique solution $\widetilde{\boldsymbol{b}}$ and there exists a unique non-empty index set $I \subseteq\{1, \ldots, d\}$ so that

$$
\begin{aligned}
& \widetilde{\boldsymbol{b}}_{I}= \boldsymbol{b}_{I} \neq \mathbf{0}_{I}, \quad M_{I I}^{-1} \boldsymbol{b}_{I}>\mathbf{0}_{I}, \\
& \text { and if } \quad I^{c}=\{1, \ldots, d\} \backslash I \neq \emptyset, \text { then } \widetilde{\boldsymbol{b}}_{I^{c}}=-\left(\left(M^{-1}\right)_{I^{c} I^{c}}\right)^{-1}\left(M^{-1}\right)_{I^{c} I} \boldsymbol{b}_{I}=M_{I^{c} I} M_{I I}^{-1} \boldsymbol{b}_{I} \geq \boldsymbol{b}_{I^{c}} .
\end{aligned}
$$

Furthermore,

$$
\begin{aligned}
\min _{\boldsymbol{x} \geq \boldsymbol{b}} \boldsymbol{x}^{\top} M^{-1} \boldsymbol{x} & =\widetilde{\boldsymbol{b}}^{\top} M^{-1} \widetilde{\boldsymbol{b}}=\boldsymbol{b}_{I}^{\top} M_{I I}^{-1} \boldsymbol{b}_{I}>0, \\
\boldsymbol{x}^{\top} M^{-1} \widetilde{\boldsymbol{b}} & =\boldsymbol{x}_{I}^{\top} M_{I I}^{-1} \widetilde{\boldsymbol{b}}_{I}=\boldsymbol{x}_{I}^{\top} M_{I I}^{-1} \boldsymbol{b}_{I}, \quad \boldsymbol{x} \in \mathbb{R}^{d} .
\end{aligned}
$$

If $\boldsymbol{b}=b \mathbf{1}, b \in(0, \infty)$, then $2 \leq \sharp\{i: i \in I\} \leq d$.

Hereafter, the unique index set $I$ that defines the solution of the quadratic programming problems in question will be referred to as the essential index set.

For any fixed $t$, let $I(t) \subseteq\{1, \ldots, d\}$ be the essential index set of the quadratic programming problem $P_{\Sigma}(\boldsymbol{b}(t))$ where

$$
\boldsymbol{b}(t)=\boldsymbol{\alpha}+t \boldsymbol{\mu}, \quad t \geq 0
$$

and set

$$
I(t)^{c}=\{1, \ldots, d\} \backslash I(t) .
$$

Next, we analyze the function $g$ defined in (6). Let us briefly mention the following standard notation for two given functions $f(\cdot)$ and $h(\cdot)$. We write $f(x)=h(x)(1+o(1))$ or simply $f(x) \sim h(x)$, if $\lim _{x \rightarrow a} f(x) / h(x)=1$ $(a \in \mathbb{R} \cup\{\infty\})$. Further, write $f(x)=o(h(x))$, if $\lim _{x \rightarrow a} f(x) / h(x)=0$.

Lemma 2.2. We have $g \in C^{1}(0, \infty)$. Furthermore, $g$ is convex and it achieves its unique minimum at

$$
t_{0}=\sqrt{\frac{\boldsymbol{\alpha}_{I}^{\top} \Sigma_{I I}^{-1} \boldsymbol{\alpha}_{I}}{\boldsymbol{\mu}_{I}^{\top} \Sigma_{I I}^{-1} \boldsymbol{\mu}_{I}}}>0,
$$

which is given by

$$
g\left(t_{0}\right)=\inf _{t>0} \frac{1}{t} \inf _{\boldsymbol{v} \geq \boldsymbol{\alpha}+\boldsymbol{\mu} t} \boldsymbol{v}^{\top} \Sigma^{-1} \boldsymbol{v}=\frac{1}{t_{0}} \boldsymbol{b}_{I}^{\top} \Sigma_{I I}^{-1} \boldsymbol{b}_{I},
$$

with

$$
\boldsymbol{b}=\boldsymbol{b}\left(t_{0}\right)=\boldsymbol{\alpha}+t_{0} \boldsymbol{\mu}
$$

and $I=I\left(t_{0}\right)$ being the essential index set corresponding to $P_{\Sigma}(\boldsymbol{b})$. Moreover,

$$
g\left(t_{0} \pm t\right)=g\left(t_{0}\right)+\frac{g^{\prime \prime}\left(t_{0} \pm\right)}{2} t^{2}(1+o(1)), \quad t \downarrow 0 .
$$


The proof of Lemma 2.2 is displayed in the Appendix.

Hereafter we shall use the notation $\boldsymbol{b}=\boldsymbol{b}\left(t_{0}\right)$, and $I=I\left(t_{0}\right)$ for the essential index set of the quadratic programming problem $P_{\Sigma}(\boldsymbol{b})$. Furthermore, let $\widetilde{\boldsymbol{b}}$ be the unique solution of $P_{\Sigma}(\boldsymbol{b})$. If $I^{c}=\{1, \ldots, d\} \backslash I \neq \emptyset$, we define the weakly essential and the unessential index sets by

$$
K=\left\{j \in I^{c}: \widetilde{\boldsymbol{b}}_{j}=\Sigma_{j I} \Sigma_{I I}^{-1} \boldsymbol{b}_{I}=\boldsymbol{b}_{j}\right\}, \quad \text { and } J=\left\{j \in I^{c}: \widetilde{\boldsymbol{b}}_{j}=\Sigma_{j I} \Sigma_{I I}^{-1} \boldsymbol{b}_{I}>\boldsymbol{b}_{j}\right\}
$$

respectively. Set for $t>0$

$$
g_{I}(t)=\frac{1}{t} \boldsymbol{\alpha}_{I}^{\top} \Sigma_{I I}^{-1} \boldsymbol{\alpha}_{I}+2 \boldsymbol{\alpha}_{I}^{\top} \Sigma_{I I}^{-1} \boldsymbol{\mu}_{I}+\boldsymbol{\mu}_{I}^{\top} \Sigma_{I I}^{-1} \boldsymbol{\mu}_{I} t
$$

Clearly, by Lemma 2.1 we have $g\left(t_{0}\right)=g_{I}\left(t_{0}\right)$. Furthermore, we have

$$
g_{I}\left(t_{0}+t\right)=g_{I}\left(t_{0}\right)+\frac{g_{I}^{\prime \prime}\left(t_{0}\right)}{2} t^{2}(1+o(1)), \quad t \rightarrow 0,
$$

with

$$
g_{I}^{\prime \prime}\left(t_{0}\right)=2 t_{0}^{-3}\left(\boldsymbol{\alpha}_{I}^{\top} \Sigma_{I I}^{-1} \boldsymbol{\alpha}_{I}\right)
$$

For notational simplicity we shall set below

$$
\widehat{g}=\inf _{t \geq 0} g(t)=g\left(t_{0}\right)=g_{I}\left(t_{0}\right), \quad \widetilde{g}=g_{I}^{\prime \prime}\left(t_{0}\right)
$$

\section{Main Results}

Let for the non-empty index set $K$ defined in (15) $\boldsymbol{Y}_{K} \stackrel{d}{\sim} \mathcal{N}\left(\mathbf{0}_{K}, D_{K K}\right)$, i.e., $\boldsymbol{Y}_{K}$ is a normally distributed random vector with mean vector $\mathbf{0}_{K}$ and covariance matrix $D_{K K}$ given by

$$
D_{K K}=\Sigma_{K K}-\Sigma_{K I} \Sigma_{I I}^{-1} \Sigma_{I K}
$$

We write $m=\sharp I:=\sharp\{i: i \in I\} \geq 1$ for the number of elements of the index set $I$. Further define the following constant

$$
\mathcal{H}_{I}=\lim _{T \rightarrow \infty} \frac{1}{T} \mathcal{H}_{I}(T), \quad \mathcal{H}_{I}(T)=\int_{\mathbb{R}^{m}} e^{\frac{1}{t_{0}} \boldsymbol{x}_{I}^{\top} \Sigma_{I I}^{-1} \boldsymbol{b}_{I}} \mathbb{P}\left\{\exists_{t \in[0, T]}(\boldsymbol{X}(t)-\boldsymbol{\mu} t)_{I}>\boldsymbol{x}_{I}\right\} d \boldsymbol{x}_{I},
$$

with respect to the essential index set $I$ and set

$$
C_{I}=\frac{1}{\sqrt{\left(2 \pi t_{0}\right)^{m}\left|\Sigma_{I I}\right|}} \int_{\mathbb{R}} e^{-\widetilde{g} \frac{x^{2}}{4}} \psi(x) d x
$$

where, for $x \in \mathbb{R}$

$$
\psi(x)=\left\{\begin{array}{cc}
1, & \text { if } K=\emptyset \\
\mathbb{P}\left\{\boldsymbol{Y}_{K}>\frac{1}{\sqrt{t_{0}}}\left(\boldsymbol{\mu}_{K}-\Sigma_{K I} \Sigma_{I I}^{-1} \boldsymbol{\mu}_{I}\right) x\right\}, & \text { if } K \neq \emptyset .
\end{array}\right.
$$

$\mathcal{H}_{I}$ 's are multidimensional counterparts of the celebrated Pickands constants, defined in the 1-dimensional setup as

$$
\lim _{T \rightarrow \infty} \frac{1}{T} \int_{\mathbb{R}} e^{x} \mathbb{P}\left\{\exists_{t \in[0, T]}\left(\sqrt{2} W_{H}(t)-t^{2 H}\right)>x\right\} d x,
$$

where $W_{H}$ is a fractional Brownian motion with Hurst parameter $H \in(0,1]$; see also [20] for the analog of $\mathcal{H}_{I}$ when $\Sigma_{I I}=I_{d}$ is the identity matrix. We refer to [10-13,15] and references therein for properties and extensions of the notion of classical Pickands constants.

The next theorem constitutes our principal result. Its proof is demonstrated in Section 4. 
Theorem 3.1. Let $\boldsymbol{\alpha}, \boldsymbol{\mu}$ satisfy (3) and let $\widehat{g}, \widetilde{g}$ be given by (17). We have as $u \rightarrow \infty$

$$
P(u) \sim C_{I} \mathcal{H}_{I} u^{\frac{1-m}{2}} e^{-\frac{\widehat{g}}{2} u}
$$

where

$$
0<\frac{t_{0}^{m-1} \boldsymbol{\mu}_{I}^{\top} \Sigma_{I I}^{-1} \boldsymbol{b}_{I}}{16 \prod_{i \in I}\left(\Sigma_{I I}^{-1} \boldsymbol{b}_{I}\right)_{i}} \leq \mathcal{H}_{I}<\infty
$$

Remark 3.2. In the case that $K=\emptyset$, direct calculations show that (20) holds with

$$
C_{I}=\frac{2^{1-m / 2} \pi^{(1-m) / 2}}{\sqrt{t_{0}^{m} \widetilde{g}\left|\Sigma_{I I}\right|}}>0 .
$$

Using the same technique as in the proof of Theorem 3.1, we can derive the approximation of the conditional passage time $\tau_{u} \mid \tau_{u}<\infty$.

Theorem 3.3. Let $\tau_{u}$ be defined in (4) and $\psi$ be defined in (19). Under the assumptions of Theorem 3.1 for any $s \in \mathbb{R}$ we have

$$
\lim _{u \rightarrow \infty} \mathbb{P}\left\{\frac{\tau_{u}-t_{0} u}{\sqrt{2 u / \widetilde{g}}} \leq s \mid \tau_{u}<\infty\right\}=\frac{\int_{-\infty}^{s} e^{-\frac{x^{2}}{2}} \psi(\sqrt{2 / \widetilde{g}}) d x}{\int_{-\infty}^{\infty} e^{-\frac{x^{2}}{2}} \psi(\sqrt{2 / \widetilde{g}} x) d x}
$$

Remark 3.4. If $K=\emptyset$, then by (19) $\frac{\tau_{u}-t_{0} u}{\sqrt{2 u / \widetilde{g}}} \mid \tau_{u}<\infty$ is asymptotically, as $u \rightarrow \infty$, approximated by a standard normal random variable.

In the rest of this section we discuss some interesting special cases and examples.

3.1. Independent components. Let $\Sigma$ be the $d \times d$ identity matrix. We focus on the case where $\boldsymbol{\alpha}>\mathbf{0}$ and

$$
\mu_{i}>0, \quad 1 \leq i \leq n, \quad \mu_{j} \leq 0, \quad n+1 \leq j \leq d
$$

is valid for some positive integer $n<d$. The result for the easier case $n=d$ will also be included. Under the above assumptions

$$
g(t)=\frac{1}{t} \inf _{\boldsymbol{v} \geq \boldsymbol{\alpha}+t \boldsymbol{\mu}} \boldsymbol{v}^{\top} \boldsymbol{v}, \quad t>0
$$

Before we state the result we need to introduce some notation. By rearranging indexes we can have the following order of constants

$$
\frac{\left|\mu_{n+1}\right|}{\alpha_{n+1}} \leq \frac{\left|\mu_{n+2}\right|}{\alpha_{n+2}} \leq \cdots \leq \frac{\left|\mu_{d}\right|}{\alpha_{d}} .
$$

Next, define $d=k_{1}>\cdots>k_{l}>k_{l+1}=n$ for which

$$
\begin{aligned}
\frac{\left|\mu_{d}\right|}{\alpha_{d}}= & \frac{\left|\mu_{j}\right|}{\alpha_{j}} ; \quad k_{2}<j \leq k_{1}=d \\
\vdots & \vdots \\
\frac{\left|\mu_{k_{l}}\right|}{\alpha_{k_{l}}}= & \frac{\left|\mu_{j}\right|}{\alpha_{j}} ; \quad n+1 \leq j \leq k_{l}
\end{aligned}
$$

and

$$
\frac{\left|\mu_{k_{l}}\right|}{\alpha_{k_{l}}}<\frac{\left|\mu_{k_{l-1}}\right|}{\alpha_{k_{l-1}}}<\cdots<\frac{\left|\mu_{k_{1}}\right|}{\alpha_{k_{1}}}
$$


implying that $0=t_{0}^{\prime}<t_{1}^{\prime}<\ldots<t_{l}^{\prime}<t_{l+1}^{\prime}=\infty$, where $t_{i}^{\prime}=\frac{\alpha_{k_{i}}}{\left|\mu_{k_{i}}\right|}, i=1, \ldots, l$ are consecutive change of dimension instants. Precisely, for the quadratic programming problem in question, constancy segments are $U_{i}=\left[t_{i-1}^{\prime}, t_{i}^{\prime}\right), i=$ $1, \ldots, l+1$, and for $t \in U_{i}$ we have $I(t)=I_{i}=\left\{1, \ldots, k_{i}\right\}$ since

$$
\boldsymbol{\alpha}_{I_{i}}+t \boldsymbol{\mu}_{I_{i}}>\mathbf{0}_{I_{i}}, \quad \boldsymbol{\alpha}_{I_{i}^{c}}+t \boldsymbol{\mu}_{I_{i}^{c}} \leq \mathbf{0}_{I_{i}^{c}}, \quad t \in U_{i}
$$

Define for $i=1, \ldots, l+1$ the following auxiliary functions

$$
g_{I_{i}}(t)=\frac{1}{t} \boldsymbol{\alpha}_{I_{i}}^{\top} \boldsymbol{\alpha}_{I_{i}}+2 \boldsymbol{\alpha}_{I_{i}}^{\top} \boldsymbol{\mu}_{I_{i}}+t \boldsymbol{\mu}_{I_{i}}^{\top} \boldsymbol{\mu}_{I_{i}}, \quad t>0
$$

and remark that $g(t)=g_{I_{i}}(t)$ for $t \in U_{i}$. Clearly, $g_{I_{i}}(t), t>0$ achieves its global minimum at

$$
t_{0}(i)=\sqrt{\frac{\boldsymbol{\alpha}_{I_{i}}^{\top} \boldsymbol{\alpha}_{I_{i}}}{\boldsymbol{\mu}_{I_{i}}^{\top} \boldsymbol{\mu}_{I_{i}}}}>0
$$

Set below

$$
p:=\min \left\{i=1, \ldots, l+1: t_{i-1}^{\prime} \leq t_{0}(i)<t_{i}^{\prime}\right\}, \quad t_{0}=t_{0}(p)=\sqrt{\frac{\boldsymbol{\alpha}_{I_{p}}^{\top} \boldsymbol{\alpha}_{I_{p}}}{\boldsymbol{\mu}_{I_{p}}^{\top} \boldsymbol{\mu}_{I_{p}}}}>0 .
$$

With the same arguments as at the end of the proof of Lemma 2.2 it follows that $g$ achieves its minimum at $t_{0}$. Then with the notation of Theorem 3.1 we have

$$
g_{I}\left(t_{0}\right)=\frac{1}{t_{0}} \sum_{j=1}^{k_{p}}\left(\alpha_{j}+\mu_{j} t_{0}\right)^{2}, \quad g_{I}^{\prime \prime}\left(t_{0}\right)=2 t_{0}^{-3} \sum_{j=1}^{k_{p}} \alpha_{j}^{2}, \quad I=I_{p}, \quad m=k_{p} .
$$

Moreover, if $t_{p-1}^{\prime}<t_{0}(p)<t_{p}^{\prime}$, then $K=\emptyset$, and if $t_{p-1}^{\prime}=t_{0}(p)$, then $K=\left\{k_{p}+1, \ldots, k_{p-1}\right\}$. Set

$$
\mathcal{H}_{I_{p}}=\lim _{T \rightarrow \infty} \frac{1}{T} \int_{\mathbb{R}^{k_{p}}} e^{\sum_{j=1}^{k_{p}}\left(\alpha_{j} t_{0}^{-1}+\mu_{j}\right) x_{j}} \mathbb{P}\left\{\exists_{t \in[0, T]}(\boldsymbol{B}(t)-\boldsymbol{\mu} t)_{I_{p}}>\boldsymbol{x}_{I_{p}}\right\} d \boldsymbol{x}_{I_{p}} .
$$

We define $\Psi(x)=1-\Phi(x), x \in \mathbb{R}$ with $\Phi$ the distribution function of an $\mathcal{N}(0,1)$ random variable. Below we shall put $\prod_{i \in \mathcal{B}}(\cdots)=1$ for $\mathcal{B}$ empty.

We reformulate next our main findings for this particular case.

Corollary 3.5. (i). If $n<d$, then as $u \rightarrow \infty$

$$
\mathbb{P}\left\{\exists_{t \geq 0} \boldsymbol{B}(t)-\boldsymbol{\mu} t>\boldsymbol{\alpha} u\right\} \sim \frac{\mathcal{H}_{I_{p}}}{\sqrt{\left(2 \pi t_{0}\right)^{k_{p}}}} u^{\frac{1-k_{p}}{2}} e^{-\frac{1}{2 t_{0}} \sum_{j=1}^{k_{p}}\left(\alpha_{j}+\mu_{j} t_{0}\right)^{2} u} \int_{\mathbb{R}} e^{-\frac{\sum_{j=1}^{k_{p}} \alpha_{j}^{2}}{2 t_{0}^{3}} x^{2}} \prod_{i \in K} \Psi\left(\frac{\mu_{i}}{\sqrt{t_{0}}} x\right) d x,
$$

and for any $s \in \mathbb{R}$

$$
\lim _{u \rightarrow \infty} \mathbb{P}\left\{\frac{\tau_{u}-t_{0} u}{\sqrt{t_{0}^{3}\left(\sum_{j=1}^{k_{p}} \alpha_{j}^{2}\right)^{-1} u}} \leq s \mid \tau_{u}<\infty\right\}=\frac{\int_{-\infty}^{s} e^{-\frac{x^{2}}{2}} \prod_{i \in K} \Psi\left(\mu_{i} t_{0}\left(\sum_{j=1}^{k_{p}} \alpha_{j}^{2}\right)^{-1 / 2} x\right) d x}{\int_{-\infty}^{\infty} e^{-\frac{x^{2}}{2}} \prod_{i \in K} \Psi\left(\mu_{i} t_{0}\left(\sum_{j=1}^{k_{p}} \alpha_{j}^{2}\right)^{-1 / 2} x\right) d x} .
$$

(ii). If $n=d$, then (23) and (24) hold with $p$ replaced by $l+1$ and $K$ replaced by $\emptyset$.

3.2. Homogeneous $\boldsymbol{\alpha}$ and $\boldsymbol{\mu}$. Suppose that $\alpha=\mathbf{1} \alpha, \alpha>0$ and $\boldsymbol{\mu}=\mathbf{1} \mu, \mu>0$. Then for any $t>0$

$$
g(t)=\frac{1}{t} \inf _{\boldsymbol{v} \geq \boldsymbol{\alpha}+\boldsymbol{\mu} t} \boldsymbol{v}^{\top} \Sigma^{-1} \boldsymbol{v}=\frac{(\alpha+\mu t)^{2}}{t} D, \quad D:=\inf _{\boldsymbol{v} \geq \mathbf{1}} \boldsymbol{v}^{\top} \Sigma^{-1} \boldsymbol{v} .
$$

Let $I$ be the essential index set of the quadratic programming problem $P_{\Sigma}(\mathbf{1})$ with $m=\sharp\{i: i \in I\}$. If $I^{c}$ is non-empty, we set

$$
K=\left\{j \in I^{c}: \Sigma_{j I} \Sigma_{I I}^{-1} \mathbf{1}_{I}=\mathbf{1}_{j}\right\}
$$

Obviously, $I(t)=I, t \geq 0$. Further, $g_{I}(t)=g(t), t>0$ and

$$
t_{0}=\alpha \mu^{-1}, \quad g_{I}\left(t_{0}\right)=4 D \alpha \mu, \quad g_{I}^{\prime \prime}\left(t_{0}\right)=2 D \mu^{3} \alpha^{-1}, \quad \boldsymbol{b}=2 \alpha \mathbf{1} .
$$


Corollary 3.6. We have, as $u \rightarrow \infty$,

$$
P(u) \sim \frac{2^{-\sharp K} \alpha^{(1-m) / 2} \mu^{(m-3) / 2}}{\sqrt{(2 \pi)^{m-1} D\left|\Sigma_{I I}\right|}} \mathcal{H}_{I} u^{\frac{1-m}{2}} e^{-2 D \alpha \mu u},
$$

and for any $s \in \mathbb{R}$

$$
\lim _{u \rightarrow \infty} \mathbb{P}\left\{\frac{\tau_{u}-\alpha \mu^{-1} u}{\sqrt{\alpha D^{-1} \mu^{-3} u}} \leq s \mid \tau_{u}<\infty\right\}=\Phi(s) .
$$

3.3. Negatively associated components. In this subsection we suppose that

$$
\Sigma^{-1} \boldsymbol{\alpha}>\mathbf{0}, \quad \Sigma^{-1} \boldsymbol{\mu}>\mathbf{0} .
$$

A special case of interest is when $\Sigma^{-1}$ has all elements positive, $\boldsymbol{\alpha}>\mathbf{0}$ and $\boldsymbol{\mu}>\mathbf{0}$. Recall that if the covariance matrix $\Sigma$ of $\boldsymbol{Z}$ is a correlation matrix, then the statement that $\Sigma^{-1}$ has all elements positive means that it is an $M$-matrix, i.e., $\Sigma=I_{d}-B$, where $B \geq 0$ and $I_{d}$ is the identity matrix. For general covariance matrix $\Sigma$, with nonpositive elements out of the diagonal, transformation $\operatorname{diag}\left(\sigma_{j j}^{-1}\right) \sum \operatorname{diag}\left(\sigma_{j j}^{-1}\right)$ makes it an $M$-matrix. Notice that if a Gaussian vector $\boldsymbol{Z}$ has such a covariance matrix, then $\boldsymbol{Z}$ is negatively associated (for definition and properties see [21]).

In this case $I=\{1, \ldots, d\}, K=J=\emptyset, m=d$ and

$$
g_{I}(t)=\frac{1}{t} \boldsymbol{\alpha}^{\top} \Sigma^{-1} \boldsymbol{\alpha}+2 \boldsymbol{\alpha}^{\top} \Sigma^{-1} \boldsymbol{\mu}+t \boldsymbol{\mu}^{\top} \Sigma^{-1} \boldsymbol{\mu} .
$$

Consequently,

$$
t_{0}=\sqrt{\frac{\boldsymbol{\alpha}^{\top} \Sigma^{-1} \boldsymbol{\alpha}}{\boldsymbol{\mu}^{\top} \Sigma^{-1} \boldsymbol{\mu}}}>0, \quad g_{I}\left(t_{0}\right)=\frac{1}{t_{0}} \boldsymbol{b}^{\top} \Sigma^{-1} \boldsymbol{b}, \quad g_{I}^{\prime \prime}\left(t_{0}\right)=\frac{2 \boldsymbol{\alpha}^{\top} \Sigma^{-1} \boldsymbol{\alpha}}{t_{0}^{3}},
$$

where $\boldsymbol{b}=\boldsymbol{\alpha}+t_{0} \boldsymbol{\mu}$. Hence we arrive at the following result.

Corollary 3.7. As $u \rightarrow \infty$

$$
P(u) \sim \frac{(2 \pi)^{(1-d) / 2} \mathcal{H}_{\{1, \ldots, d\}}}{\sqrt{t_{0}^{d-3}\left(\boldsymbol{\alpha}^{\top} \Sigma^{-1} \boldsymbol{\alpha}\right)|\Sigma|}} u^{\frac{1-d}{2}} e^{-\frac{\boldsymbol{b}^{\top} \Sigma^{-1} b}{2 t_{0}} u},
$$

and for any $s \in \mathbb{R}$

$$
\lim _{u \rightarrow \infty} \mathbb{P}\left\{\frac{\tau_{u}-t_{0} u}{\sqrt{t_{0}^{3}\left(\boldsymbol{\alpha}^{\top} \Sigma^{-1} \boldsymbol{\alpha}\right)^{-1} u}} \leq s \mid \tau_{u}<\infty\right\}=\Phi(s) .
$$

3.4. Two-dimensional case. In this section we analyze some interesting scenarios of the two-dimensional case, in which we can observe how different entries of the covariance matrix yield different scenarios of asymptotic behaviour. Proofs will be postponed to Section 5.3, after presenting required results on a quadratic programming problem. For simplicity, we shall assume that

$$
\Sigma=\left(\begin{array}{ll}
1 & \rho \\
\rho & 1
\end{array}\right), \quad \rho \in(-1,1)
$$

and $\mu_{i}=1, i=1,2, \alpha_{1}>\alpha_{2}>0$.

We present next the asymptotics of (2) for the 2-dimensional model. 
Corollary 3.8. (i). If $-1<\rho<\frac{\alpha_{1}+\alpha_{2}}{2 \alpha_{1}}$, then as $u \rightarrow \infty$

$$
P(u) \sim \frac{\mathcal{H}_{\{1,2\}}}{\sqrt{t_{0}^{2} \pi\left(1-\rho^{2}\right) \widetilde{g}}} u^{-\frac{1}{2}} e^{-\frac{\widehat{g}}{2} u}
$$

with

$$
\begin{aligned}
& t_{0}=\sqrt{\frac{\alpha_{1}^{2}+\alpha_{2}^{2}-2 \alpha_{1} \alpha_{2} \rho}{2(1-\rho)}}>0, \quad \widehat{g}=\frac{2}{1+\rho}\left(\alpha_{1}+\alpha_{2}+2 t_{0}\right), \quad \widetilde{g}=2 t_{0}^{-3} \frac{\alpha_{1}^{2}+\alpha_{2}^{2}-2 \alpha_{1} \alpha_{2} \rho}{1-\rho^{2}}, \\
& \mathcal{H}_{\{1,2\}}=\lim _{T \rightarrow \infty} \frac{1}{T} \int_{\mathbb{R}^{2}} e^{\left(\frac{\alpha_{1}-\rho \alpha_{2}}{\left(1-\rho^{2}\right) t_{0}}+\frac{1}{1+\rho}\right) x_{1}+\left(\frac{\alpha_{2}-\rho \alpha_{1}}{\left(1-\rho^{2}\right) t_{0}}+\frac{1}{1+\rho}\right) x_{2}} \mathbb{P}\left\{\exists_{t \in[0, T]}\left(\boldsymbol{X}(t)-t(1,1)^{T}\right)>\boldsymbol{x}\right\} d \boldsymbol{x} .
\end{aligned}
$$

Furthermore, for any $s \in \mathbb{R}$

$$
\lim _{u \rightarrow \infty} \mathbb{P}\left\{\frac{\tau_{u}-t_{0} u}{\sqrt{2 u / \widetilde{g}}} \leq s \mid \tau_{u}<\infty\right\}=\Phi(s)
$$

(ii). If $\rho=\frac{\alpha_{1}+\alpha_{2}}{2 \alpha_{1}}$, then as $u \rightarrow \infty$

$$
P(u) \sim \frac{1}{\sqrt{2 \pi \alpha_{1}}} \int_{\mathbb{R}} e^{-\frac{1}{2 \alpha_{1}} x^{2}} \Psi\left(\frac{1-\rho}{\sqrt{\alpha_{1}}} x\right) d x e^{-2 \alpha_{1} u},
$$

and for any $s \in \mathbb{R}$

$$
\lim _{u \rightarrow \infty} \mathbb{P}\left\{\frac{\tau_{u}-\alpha_{1} u}{\sqrt{\alpha_{1} u}} \leq s \mid \tau_{u}<\infty\right\}=\frac{\int_{-\infty}^{s} e^{-\frac{x^{2}}{2}} \Psi((1-\rho) x) d x}{\int_{-\infty}^{\infty} e^{-\frac{x^{2}}{2}} \Psi((1-\rho) x) d x} .
$$

(iii). If $\frac{\alpha_{1}+\alpha_{2}}{2 \alpha_{1}}<\rho<1$, then as $u \rightarrow \infty$

$$
P(u) \sim e^{-2 \alpha_{1} u}
$$

and for any $s \in \mathbb{R}$

$$
\lim _{u \rightarrow \infty} \mathbb{P}\left\{\alpha_{1}^{-1 / 2}\left(\tau_{u}-\alpha_{1} u\right) / \sqrt{u} \leq s \mid \tau_{u}<\infty\right\}=\Phi(s)
$$

Remark 3.9. According to our findings, in both (ii) and (iii) above we should also have the following constant

$$
\mathcal{H}_{\{1\}}=\lim _{T \rightarrow \infty} \frac{1}{T} \int_{\mathbb{R}} e^{2 x} \mathbb{P}\left\{\exists_{t \in[0, T]}(\boldsymbol{X}(t)-t)_{1}>x\right\} d x .
$$

However, a simple comparison with the known Pickands constants for the standard Brownian motion, i.e.,

$$
\lim _{T \rightarrow \infty} \frac{1}{T} \int_{\mathbb{R}} e^{x} \mathbb{P}\left\{\sup _{t \in[0, T]}\left(\sqrt{2} B_{1}(t)-t\right)>x\right\} d x=1
$$

yields $\mathcal{H}_{\{1\}}=1$.

We conclude this section with some observations.

It is possible to have similar asymptotics of $P(u)$ for $d \geq 2$ as in the 1-dimensional case. For instance in the above the 2-dimensional setup, for $\rho \in\left(\left(\alpha_{1}+\alpha_{2}\right) /\left(2 \alpha_{1}\right), 1\right)$ we have

$$
\mathbb{P}\left\{\exists_{t \geq 0} \cap_{i=1}^{2}\left\{\left(X_{i}(t)-t\right)>\alpha_{i} u\right\}\right\} \sim C_{I} \mathbb{P}\left\{\exists_{t \geq 0}\left(X_{1}(t)-t\right)>\alpha_{1} u\right\}, \quad u \rightarrow \infty,
$$

with $C_{I}=1$. Consequently, only the first component of $\boldsymbol{X}(t), t \geq 0$ is controlling the asymptotics of $P(u)$. This case will be referred to as the loss of dimensions phenomena.

There are other cases of loss of dimensions phenomena, where some components other than those with indexes in $I$ still play a role in the asymptotics of $P(u)$, but only up to some constants. For instance, referring again to the 
2-dimensional case presented in Corollary 3.8 we have for $\rho=\left(\alpha_{1}+\alpha_{2}\right) /\left(2 \alpha_{1}\right)$ that $(25)$ holds, with $C_{I}$ taking the information of the second component and given by

$$
C_{I}=\frac{1}{\sqrt{2 \pi \alpha_{1}}} \int_{\mathbb{R}} e^{-\frac{1}{2 \alpha_{1}} x^{2}} \Psi\left(\frac{1-\rho}{\sqrt{\alpha_{1}}} x\right) d x .
$$

There are several technical issues related to the loss of dimensions as it will be explained in our proofs below.

\section{Proofs of Main Results}

In this section we first present the proof of Theorem 3.1. In order to convey the main ideas and to reduce complexity, we shall divide the proof into several steps and then we complete the proof by putting all the arguments together. By the self-similarity of Brownian motion, for any $u$ positive we have

$$
P(u)=\mathbb{P}\left\{\exists_{t \geq 0} \quad \boldsymbol{X}(t)-\boldsymbol{\mu} t>\boldsymbol{\alpha} u\right\}=\mathbb{P}\left\{\exists_{t \geq 0} \quad \boldsymbol{X}(t)>\sqrt{u}(\boldsymbol{\alpha}+\boldsymbol{\mu} t)\right\} .
$$

We have thus the following sandwich bounds

$$
p(u) \leq P(u) \leq p(u)+r(u)
$$

where

$$
p(u):=\mathbb{P}\left\{\exists_{t \in \triangle_{u}} \boldsymbol{X}(t)>\sqrt{u}(\boldsymbol{\alpha}+\boldsymbol{\mu} t)\right\}, \quad r(u):=\mathbb{P}\left\{\exists_{t \in \widetilde{\triangle}_{u}} \boldsymbol{X}(t)>\sqrt{u}(\boldsymbol{\alpha}+\boldsymbol{\mu} t)\right\}
$$

with (recall the definition of $t_{0}$ in $(12)$ )

$$
\triangle_{u}=\left[t_{0}-\frac{\ln (u)}{\sqrt{u}}, t_{0}+\frac{\ln (u)}{\sqrt{u}}\right], \quad \widetilde{\triangle}_{u}=\left[0, t_{0}-\frac{\ln (u)}{\sqrt{u}}\right] \cup\left[t_{0}+\frac{\ln (u)}{\sqrt{u}}, \infty\right) .
$$

4.1. Analysis of $r(u)$. This step is concerned with sharp upper bounds for $r(u)$ when $u$ is large.

Lemma 4.1. For all large $u$ we have

$$
\mathbb{P}\left\{\exists_{t \in\left[t_{0}+\frac{\ln (u)}{\sqrt{u}}, \infty\right)} \boldsymbol{X}(t)>\sqrt{u}(\boldsymbol{\alpha}+\boldsymbol{\mu} t)\right\} \leq C e^{-\frac{u}{2} g_{I}\left(t_{0}\right)-\left(\frac{g^{\prime \prime}\left(t_{0}+\right)}{2}-\varepsilon\right)(\ln (u))^{2}}
$$

and

$$
\mathbb{P}\left\{\exists_{t \in\left[0, t_{0}-\frac{\ln (u)}{\sqrt{u}}\right]} \boldsymbol{X}(t)>\sqrt{u}(\boldsymbol{\alpha}+\boldsymbol{\mu} t)\right\} \leq C e^{-\frac{u}{2} g_{I}\left(t_{0}\right)-\left(\frac{g^{\prime \prime}\left(t_{0}-\right)}{2}-\varepsilon\right)(\ln (u))^{2}}
$$

are valid for some constant $C>0$ and some sufficiently small $\varepsilon>0$ which do not depend on $u$.

Proof: We only present the proof of (27) since the proof of (28) follows with similar arguments. First note that for any $D \subset \mathbb{R}_{+}$and any $u$ positive

$$
\begin{aligned}
& \mathbb{P}\left\{\exists_{t \in D} \boldsymbol{X}(t)>\sqrt{u}(\boldsymbol{\alpha}+\boldsymbol{\mu} t)\right\} \\
& \leq \mathbb{P}\left\{\exists_{t \in D}(\boldsymbol{X}(t))_{I(t)}>\sqrt{u}(\boldsymbol{\alpha}+\boldsymbol{\mu} t)_{I(t)}\right\} \\
& \leq \mathbb{P}\left\{\exists_{t \in D}\left(\Sigma_{I(t) I(t)}^{-1}(\boldsymbol{\alpha}+\boldsymbol{\mu} t)_{I(t)}\right)^{\top}(\boldsymbol{X}(t))_{I(t)}>\sqrt{u}\left(\Sigma_{I(t) I(t)}^{-1}(\boldsymbol{\alpha}+\boldsymbol{\mu} t)_{I(t)}\right)^{\top}(\boldsymbol{\alpha}+\boldsymbol{\mu} t)_{I(t)}\right\} \\
& =\mathbb{P}\left\{\exists_{t \in D} Y_{I(t)}(t)>\sqrt{u}\right\},
\end{aligned}
$$

where we used the fact that $\Sigma_{I(t) I(t)}^{-1}(\boldsymbol{\alpha}+\boldsymbol{\mu} t)_{I(t)}>\mathbf{0}_{I(t)}$ for all $t \geq 0$, and

$$
Y_{I(t)}(t)=\frac{\left(\Sigma_{I(t) I(t)}^{-1}(\boldsymbol{\alpha}+\boldsymbol{\mu} t)_{I(t)}\right)^{\top}(\boldsymbol{X}(t))_{I(t)}}{(\boldsymbol{\alpha}+\boldsymbol{\mu} t)_{I(t)}^{\top} \Sigma_{I(t) I(t)}^{-1}(\boldsymbol{\alpha}+\boldsymbol{\mu} t)_{I(t)}}, \quad t \geq 0
$$


By the property of Brownian motion, we have almost surely

$$
\lim _{t \rightarrow \infty} Y_{I(t)}(t)=0
$$

inplying that $Y_{I(t)}$ has bounded sample paths on $[a, \infty)$ for any $a>0$. Since further

$$
\operatorname{Var}\left(Y_{I(t)}(t)\right)=\frac{1}{g(t)}, \quad t \geq 0
$$

by the Borell-TIS inequality (see e.g., [22-25]) for any small $\theta>0$

$$
\begin{aligned}
\mathbb{P}\left\{\exists_{t \in\left[t_{0}+\theta, \infty\right)} \boldsymbol{X}(t)>\sqrt{u}(\boldsymbol{\alpha}+\boldsymbol{\mu} t)\right\} & \leq \mathbb{P}\left\{\exists_{t \in\left[t_{0}+\theta, \infty\right)} Y_{I(t)}(t)>\sqrt{u}\right\} \\
& \leq e^{-\frac{\left(\sqrt{u}-C_{0}\right)^{2}}{2} \inf _{t \in\left[t_{0}+\theta, \infty\right)} g(t)}
\end{aligned}
$$

holds for all $u$ such that

$$
\sqrt{u}>C_{0}:=\mathbb{E}\left\{\sup _{t \in\left[t_{0}+\theta, \infty\right)} Y_{I(t)}(t)\right\}
$$

It follows from Lemma 5.4 that if $\theta>0$ is chosen sufficiently small, then for some $I^{+}$

$$
g(t)=\frac{1}{t} \boldsymbol{\alpha}_{I^{+}}^{\top} \Sigma_{I^{+} I^{+}}^{-1} \boldsymbol{\alpha}_{I^{+}}+2 \boldsymbol{\alpha}_{I^{+}}^{\top} \Sigma_{I^{+} I^{+}}^{-1} \boldsymbol{\mu}_{I^{+}}+\boldsymbol{\mu}_{I^{+}}^{\top} \Sigma_{I^{+} I^{+}}^{-1} \boldsymbol{\mu}_{I^{+}} t
$$

for all $t \in\left(t_{0}, t_{0}+\theta\right)$. Furthermore,

$$
\mathbb{E}\left\{\left(Y_{I^{+}}(t)-Y_{I^{+}}(s)\right)^{2}\right\} \leq C_{1}|t-s|
$$

holds for all $s, t \in\left[t_{0}+\frac{\ln (u)}{\sqrt{u}}, t_{0}+\theta\right]$, with some positive constant $C_{1}$. Thus, it follows from Piterbarg's inequality in [26][Lemma 5.1] (see also [12][Theorem 8.1] and [27][Theorem 3]) that

$$
\begin{aligned}
\mathbb{P}\left\{\exists_{t \in\left[t_{0}+\frac{\ln (u)}{\sqrt{u}}, t_{0}+\theta\right]} \boldsymbol{X}(t)>\sqrt{u}(\boldsymbol{\alpha}+\boldsymbol{\mu} t)\right\} & \leq \mathbb{P}\left\{\exists_{t \in\left[t_{0}+\frac{\ln (u)}{\sqrt{u}}, t_{0}+\theta\right]} Y_{I^{+}}(t)>\sqrt{u}\right\} \\
& \leq C_{2} u e^{-\frac{u}{2} \inf _{t \in\left[t_{0}+\frac{\ln (u)}{\sqrt{u}}, t_{0}+\theta\right]} g(t)}
\end{aligned}
$$

holds for all $u$ large, with some positive constant $C_{2}$ not depending on $u$. Moreover, for a small chosen $\theta>0$, there exists some $\varepsilon>0$ such that

$$
\inf _{t \in\left[t_{0}+\frac{\ln (u)}{\sqrt{u}}, t_{0}+\theta\right]} g(t) \geq g\left(t_{0}\right)+\left(\frac{g^{\prime \prime}\left(t_{0}+\right)}{2}-\varepsilon\right) \frac{(\ln (u))^{2}}{u}
$$

is valid for all $u$ large with

$$
g^{\prime \prime}\left(t_{0}+\right)=2 t_{0}^{-3}\left(\boldsymbol{\alpha}_{I^{+}}^{\top} \Sigma_{I^{+} I^{+}}^{-1} \boldsymbol{\alpha}_{I^{+}}\right)>0
$$

Consequently, the claim in (27) follows by (29), (30), (31) and the fact that

$$
g\left(t_{0}\right)=g_{I}\left(t_{0}\right)<\inf _{t \in\left[t_{0}+\theta, \infty\right)} g(t)
$$

Hence the proof is complete. 
4.2. Analysis of $p(u)$. We investigate the asymptotics of $p(u)$ as $u \rightarrow \infty$. Denote, for any fixed $T>0$ and $u>0$

$$
\triangle_{j ; u}=\triangle_{j ; u}(T)=\left[t_{0}+j T u^{-1}, t_{0}+(j+1) T u^{-1}\right], \quad-N_{u} \leq j \leq N_{u}
$$

where $N_{u}=\left\lfloor T^{-1} \ln (u) \sqrt{u}\right\rfloor$ (we denote by $\lfloor\cdot\rfloor$ the ceiling function). By Bonferroni's inequality we have

$$
p_{1}(u) \geq p(u) \geq p_{2}(u)-\Pi(u)
$$

where

$$
p_{1}(u)=\sum_{j=-N_{u}-1}^{N_{u}} p_{j ; u}, \quad p_{2}(u)=\sum_{j=-N_{u}}^{N_{u}-1} p_{j ; u}, \quad \Pi(u)=\sum_{-N_{u} \leq j<l \leq N_{u}} p_{i, j ; u}
$$

with

$$
p_{j ; u}=\mathbb{P}\left\{\exists_{t \in \Delta_{j ; u}} \boldsymbol{X}(t)>\sqrt{u}(\boldsymbol{\alpha}+t \boldsymbol{\mu})\right\}
$$

and

$$
p_{i, j ; u}=\mathbb{P}\left\{\exists_{t \in \triangle_{i ; u}} \boldsymbol{X}(t)>\sqrt{u}(\boldsymbol{\alpha}+\boldsymbol{\mu} t), \exists_{t \in \triangle_{j ; u}} \boldsymbol{X}(t)>\sqrt{u}(\boldsymbol{\alpha}+\boldsymbol{\mu} t)\right\}
$$

Analysis of the single sum. We shall focus on the asymptotics of $p_{1}(u)$, which will be easily seen to be asymptotically equivalent to $p_{2}(u)$ as $u \rightarrow \infty$.

We first present a lemma concerning the finiteness of $\mathcal{H}_{I}(T)$ defined in (18), the constant that will appear in the asymptotics of $p_{1}(u)$.

Lemma 4.2. For any $T>0$ we have that $\mathcal{H}_{I}(T)<\infty$.

Proof: The claim follows if we can show that for any $\boldsymbol{a}_{I}>\mathbf{0}_{I}$ and any $T>0$ we have

$$
\int_{\mathbb{R}^{m}} e^{\boldsymbol{x}_{I}^{\top} \boldsymbol{a}_{I}} \mathbb{P}\left\{\exists_{t \in[0, T]}(\boldsymbol{X}(t)-\boldsymbol{\mu} t)_{I}>\boldsymbol{x}_{I}\right\} d \boldsymbol{x}_{I}<\infty .
$$

Clearly, it is sufficient to prove that

$$
\int_{\left|\boldsymbol{x}_{I}\right|>L \mathbf{1}_{I}} e^{\boldsymbol{x}_{I}^{\top} \boldsymbol{a}_{I}} \mathbb{P}\left\{\exists_{t \in[0, T]}(\boldsymbol{X}(t)-\boldsymbol{\mu} t)_{I}>\boldsymbol{x}_{I}\right\} d \boldsymbol{x}_{I}<\infty
$$

holds for some large $L$. Obviously, the above integral is the sum of a finite number of integrals with $\boldsymbol{x}_{I}$ restricted to certain quadrants. Thus, without loss of generality, we may consider only the integral over $\left\{\boldsymbol{x}_{I_{1}}>L \mathbf{1}_{I_{1}}, \boldsymbol{x}_{I_{2}}<\right.$ $\left.-L \mathbf{1}_{I_{2}}\right\}$ with $I_{1} \cup I_{2}=I$. By Borell-TIS inequality

$$
\begin{aligned}
& \mathbb{P}\left\{\exists_{t \in[0, T]}(\boldsymbol{X}(t)-\boldsymbol{\mu} t)_{I}>\boldsymbol{x}_{I}\right\} \leq \mathbb{P}\left\{\exists_{t \in[0, T]}(\boldsymbol{X}(t)-\boldsymbol{\mu} t)_{I_{1}}>\boldsymbol{x}_{I_{1}}\right\} \\
& \quad \leq \mathbb{P}\left\{\exists_{t \in[0, T]} \Sigma_{i \in I_{1}}\left(X_{i}(t)-\mu_{i} t\right) \geq \Sigma_{i \in I_{1}} x_{i}\right\} \leq \exp \left(-\mathcal{C}_{1}\left(\sum_{i \in I_{1}} x_{i}-\mathcal{C}_{2}\right)^{2}\right)
\end{aligned}
$$

holds for all $L$ large enough, with some positive constants $\mathcal{C}_{1}, \mathcal{C}_{2}$ which may depend on $T$, $\boldsymbol{\mu}$. Consequently, we may further write

$$
\begin{aligned}
& \int_{\left\{\boldsymbol{x}_{I_{1}}>L \mathbf{1}_{I_{1}}, \boldsymbol{x}_{I_{2}} \leq-L \mathbf{1}_{I_{2}}\right\}} e^{\boldsymbol{x}_{I}^{\top} \boldsymbol{a}_{I}} \mathbb{P}\left\{\exists_{t \in[0, T]}(\boldsymbol{X}(t)-\boldsymbol{\mu} t)_{I}>\boldsymbol{x}_{I}\right\} d \boldsymbol{x}_{I} \\
& \leq \int_{\boldsymbol{x}_{I_{1}}>L \mathbf{1}_{I_{1}}} e^{\boldsymbol{x}_{I_{1}}^{\top} \boldsymbol{a}_{I_{1}}} \exp \left(-\mathcal{C}_{1}\left(\sum_{i \in I_{1}} x_{i}-\mathcal{C}_{2}\right)^{2}\right) d \boldsymbol{x}_{I_{1}} \int_{\boldsymbol{x}_{I_{2}}<-L \mathbf{1}_{I_{2}}} e^{\boldsymbol{x}_{I_{2}}^{\top} \boldsymbol{a}_{I_{2}}} d \boldsymbol{x}_{I_{2}}<\infty
\end{aligned}
$$

establishing thus the claim. 
Lemma 4.3. We have as $u \rightarrow \infty$

$$
p_{1}(u) \sim p_{2}(u) \sim \frac{1}{\sqrt{\left(2 \pi t_{0}\right)^{m}\left|\Sigma_{I I}\right|}} \frac{\mathcal{H}_{I}(T)}{T} u^{\frac{1-m}{2}} e^{-\frac{u}{2} g_{I}\left(t_{0}\right)} \int_{\mathbb{R}} e^{-\frac{g_{I}^{\prime \prime}\left(t_{0}\right) x^{2}}{4}} \psi(x) d x,
$$

where $\psi(x)$ is given in (19).

Proof: By the independence of the increments property and the self-similarity of the Brownian motion, we have

$$
B_{i}\left(t u^{-1}+c_{j ; u}\right) \stackrel{d}{=} \sqrt{c_{j ; u}} N_{i}+\frac{1}{\sqrt{u}} B_{i}(t), t \in[0, T], \quad i=1, \ldots, d,
$$

with $c_{j ; u}=c_{j ; u}(T)=t_{0}+j T / u$, and $\boldsymbol{N}=\left(N_{1}, \ldots, N_{d}\right)$ with independent $\mathcal{N}(0,1)$ components, being further independent of $\boldsymbol{B}$. Denote $\boldsymbol{Z}_{j ; u}=\sqrt{c_{j ; u}} A \boldsymbol{N}$ with covariance matrix $\Sigma_{j ; u}=c_{j ; u} \Sigma$ and set

$$
\boldsymbol{b}_{j ; u}=\boldsymbol{b}_{j ; u}(T)=\boldsymbol{b}\left(t_{0}+\frac{j T}{u}\right)=\boldsymbol{b}+\frac{j T}{u} \boldsymbol{\mu} .
$$

Using (35) we obtain

$$
\begin{aligned}
p_{j ; u} & =\mathbb{P}\left\{\exists_{t \in\left[t_{0}+\frac{j T}{u}, t_{0}+\frac{(j+1) T}{u}\right]} \boldsymbol{X}\left(t_{0}+\frac{j T}{u}\right)+\boldsymbol{X}(t)-\boldsymbol{X}\left(t_{0}+\frac{j T}{u}\right)>\sqrt{u}(\boldsymbol{\alpha}+t \boldsymbol{\mu})\right\} \\
& =\mathbb{P}\left\{\exists_{s \in\left[0, \frac{T}{u}\right]} \sqrt{c_{j ; u}} A \boldsymbol{N}+\boldsymbol{X}(s)>\sqrt{u}\left(\boldsymbol{\alpha}+\left(s+t_{0}+\frac{j T}{u}\right) \boldsymbol{\mu}\right)\right\} \\
& =\mathbb{P}\left\{\exists_{t \in[0, T]} \sqrt{c_{j ; u}} A \boldsymbol{N}+\frac{1}{\sqrt{u}}(\boldsymbol{X}(t)-t \boldsymbol{\mu})>\sqrt{u}\left(\boldsymbol{b}+\frac{j T}{u} \boldsymbol{\mu}\right)\right\} \\
& =\mathbb{P}\left\{\exists_{t \in[0, T]} \boldsymbol{Z}_{j ; u}+\frac{1}{\sqrt{u}}(\boldsymbol{X}(t)-t \boldsymbol{\mu})>\sqrt{u} \boldsymbol{b}_{j ; u}\right\} .
\end{aligned}
$$

Since further

$$
\begin{aligned}
& \left(\boldsymbol{Z}_{j ; u}\right)_{I} \stackrel{d}{\sim} \mathcal{N}\left(\mathbf{0}_{I},\left(\Sigma_{j ; u}\right)_{I I}\right) \\
& \left(\boldsymbol{Z}_{j ; u}\right)_{I^{c}} \mid\left(\left(\boldsymbol{Z}_{j ; u}\right)_{I}=\boldsymbol{w}_{I}\right) \stackrel{d}{\sim} \mathcal{N}\left(\Sigma_{I^{c}, I} \Sigma_{I I}^{-1} \boldsymbol{w}_{I},\left(C_{j T, u}\right)_{I^{c}}\right),
\end{aligned}
$$

with $\left(C_{j T, u}\right)_{I^{c}}=c_{j ; u}\left(\Sigma_{I^{c}, I^{c}}-\Sigma_{I^{c}, I} \Sigma_{I I}^{-1} \Sigma_{I, I^{c}}\right)$, we have

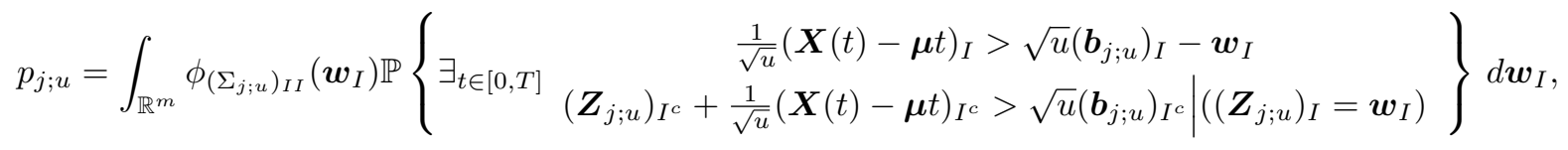

where

$$
\phi_{\left(\Sigma_{j ; u}\right)_{I I}}\left(\boldsymbol{w}_{I}\right)=\frac{1}{\sqrt{(2 \pi)^{m}\left|\left(\Sigma_{j ; u}\right)_{I I}\right|}} \exp \left(-\frac{1}{2} \boldsymbol{w}_{I}^{\top}\left(\Sigma_{j ; u}\right)_{I I}^{-1} \boldsymbol{w}_{I}\right) .
$$

Using a change of variable $\boldsymbol{w}_{I}=\sqrt{u}\left(\boldsymbol{b}_{j ; u}\right)_{I}-\boldsymbol{x}_{I} / \sqrt{u}$ we obtain

$$
\begin{aligned}
& p_{j ; u}=\frac{u^{-m / 2}}{\sqrt{(2 \pi)^{m}\left|\left(\Sigma_{j ; u}\right)_{I I}\right|}} \int_{\mathbb{R}^{m}} \exp \left(-\frac{1}{2}\left(\sqrt{u}\left(\boldsymbol{b}_{j ; u}\right)_{I}-\boldsymbol{x}_{I} / \sqrt{u}\right)^{\top}\left(\Sigma_{j ; u}\right)_{I I}^{-1}\left(\sqrt{u}\left(\boldsymbol{b}_{j ; u}\right)_{I}-\boldsymbol{x}_{I} / \sqrt{u}\right)\right) \\
& \times \mathbb{P}\left\{\begin{array}{c}
(\boldsymbol{X}(t)-\boldsymbol{\mu} t)_{I}>\boldsymbol{x}_{I} \\
\exists_{t \in[0, T]} \\
\sqrt{c_{j ; u}} \boldsymbol{Y}_{I^{c}}+\Sigma_{I^{c}, I} \Sigma_{I I}^{-1}\left(\sqrt{u}\left(\boldsymbol{b}_{j ; u}\right)_{I}-\boldsymbol{x}_{I} / \sqrt{u}\right)+\frac{1}{\sqrt{u}}(\boldsymbol{X}(t)-\boldsymbol{\mu} t)_{I^{c}}>\sqrt{u}\left(\boldsymbol{b}_{j ; u}\right)_{I^{c}}
\end{array}\right\} d \boldsymbol{x}_{I},
\end{aligned}
$$

where

$$
\boldsymbol{Y}_{I^{c}} \stackrel{d}{\sim} \mathcal{N}\left(\mathbf{0}_{I^{c}}, D_{I^{c} I^{c}}\right), \quad D_{I^{c} I^{c}}=\Sigma_{I^{c} I^{c}}-\Sigma_{I^{c} I} \Sigma_{I I}^{-1} \Sigma_{I I^{c}}
$$

Next, we work out the exponent under the above integral

$$
\left(\sqrt{u}\left(\boldsymbol{b}_{j ; u}\right)_{I}-\boldsymbol{x}_{I} / \sqrt{u}\right)^{\top}\left(\Sigma_{j ; u}\right)_{I I}^{-1}\left(\sqrt{u}\left(\boldsymbol{b}_{j ; u}\right)_{I}-\boldsymbol{x}_{I} / \sqrt{u}\right)
$$




$$
\begin{aligned}
& =u \frac{1}{c_{j ; u}}\left(\boldsymbol{b}_{j ; u}\right)_{I}^{\top} \Sigma_{I I}^{-1}\left(\boldsymbol{b}_{j ; u}\right)_{I}-2 \frac{1}{c_{j ; u}} \boldsymbol{x}_{I}^{\top} \Sigma_{I I}^{-1}\left(\boldsymbol{b}_{j ; u}\right)_{I}+\frac{1}{u c_{j ; u}} \boldsymbol{x}_{I}^{\top} \Sigma_{I I}^{-1} \boldsymbol{x}_{I} \\
& =u g_{I}\left(t_{0}+\frac{j T}{u}\right)-2 \frac{1}{c_{j ; u}} \boldsymbol{x}_{I}^{\top} \Sigma_{I I}^{-1}\left(\boldsymbol{b}_{j ; u}\right)_{I}+\frac{1}{u c_{j ; u}} \boldsymbol{x}_{I}^{\top} \Sigma_{I I}^{-1} \boldsymbol{x}_{I} .
\end{aligned}
$$

Note that

$$
\begin{aligned}
\sqrt{u}\left(\boldsymbol{b}_{j ; u}\right)_{I^{c}} & =\sqrt{u} \boldsymbol{b}_{I^{c}}+\boldsymbol{\mu}_{I^{c}} \frac{j T}{\sqrt{u}} \\
\sqrt{u} \Sigma_{I^{c}, I} \Sigma_{I I}^{-1}\left(\boldsymbol{b}_{j ; u}\right)_{I} & =\sqrt{u} \Sigma_{I^{c}, I} \Sigma_{I I}^{-1} \boldsymbol{b}_{I}+\frac{j T}{\sqrt{u}} \Sigma_{I^{c}, I} \Sigma_{I I}^{-1} \boldsymbol{\mu}_{I} .
\end{aligned}
$$

Furthermore, denote

$$
\begin{aligned}
\boldsymbol{Z}_{K}\left(t, \boldsymbol{x}_{I}\right) & =(\boldsymbol{X}(t)-t \boldsymbol{\mu})_{K}-\Sigma_{K I} \Sigma_{I I}^{-1} \boldsymbol{x}_{I}, \\
\boldsymbol{Z}_{J}\left(t, \boldsymbol{x}_{I}\right) & =(\boldsymbol{X}(t)-t \boldsymbol{\mu})_{J}-\Sigma_{J I} \Sigma_{I I}^{-1} \boldsymbol{x}_{I} .
\end{aligned}
$$

For any $u$ positive we have

$$
\begin{aligned}
& \left\{\sqrt{c_{j ; u}} Y_{I^{c}}+\Sigma_{I^{c}, I} \Sigma_{I I}^{-1}\left(\sqrt{u}\left(\boldsymbol{b}_{j ; u}\right)_{I}-\frac{\boldsymbol{x}_{I}}{\sqrt{u}}\right)+\frac{1}{\sqrt{u}}(\boldsymbol{X}(t)-t \boldsymbol{\mu})_{I^{c}}>\sqrt{u}\left(\boldsymbol{b}_{j ; u}\right)_{I^{c}}\right\} \\
& =\left\{\begin{array}{l}
\sqrt{c_{j ; u}} Y_{K}+\frac{1}{\sqrt{u}} \boldsymbol{Z}_{K}\left(t, \boldsymbol{x}_{I}\right)>\frac{j T}{\sqrt{u}}\left(\boldsymbol{\mu}_{K}-\Sigma_{K I} \Sigma_{I I}^{-1} \boldsymbol{\mu}_{I}\right) \\
\sqrt{c_{j ; u}} Y_{J}+\frac{1}{\sqrt{u}} \boldsymbol{Z}_{J}\left(t, \boldsymbol{x}_{I}\right)>\sqrt{u}\left(\boldsymbol{b}_{J}-\Sigma_{J I} \Sigma_{I I}^{-1} \boldsymbol{b}_{I}+\left(\boldsymbol{\mu}_{J}-\Sigma_{J I} \Sigma_{I I}^{-1} \boldsymbol{\mu}_{I}\right) \frac{j T}{u}\right)
\end{array}\right\},
\end{aligned}
$$

where we used $\boldsymbol{b}_{K}-\Sigma_{K I} \Sigma_{I I}^{-1} \boldsymbol{b}_{I}=\mathbf{0}_{K}$. Consequently, for the single sum we have

$$
\begin{aligned}
p_{1}(u) & =\frac{u^{-m / 2}}{\sqrt{(2 \pi)^{m}\left|\Sigma_{I I}\right|}} \sum_{-N_{u}-1 \leq j \leq N_{u}} \frac{1}{c_{j ; u}^{m / 2}} \exp \left(-\frac{1}{2} u g_{I}\left(t_{0}+\frac{j T}{u}\right)\right) \int_{\mathbb{R}^{m}} f_{j ; u}\left(T, \boldsymbol{x}_{I}\right) P_{j ; u}\left(T, \boldsymbol{x}_{I}\right) d \boldsymbol{x}_{I} \\
& =: \frac{1}{T} \frac{1}{\sqrt{(2 \pi)^{m}\left|\Sigma_{I I}\right|}} u^{(1-m) / 2} e^{-\frac{u g_{I}\left(t_{0}\right)}{2}} R_{T}(u),
\end{aligned}
$$

where

$$
\begin{aligned}
& f_{j ; u}\left(T, \boldsymbol{x}_{I}\right)= \exp \left(\frac{1}{c_{j ; u}} \boldsymbol{x}_{I}^{\top} \Sigma_{I I}^{-1}\left(\boldsymbol{b}_{j ; u}\right)_{I}-\frac{1}{2 u c_{j ; u}} \boldsymbol{x}_{I}^{\top} \Sigma_{I I}^{-1} \boldsymbol{x}_{I}\right), \\
& P_{j ; u}\left(T, \boldsymbol{x}_{I}\right)=\mathbb{P}\left\{\begin{array}{ll}
(\boldsymbol{X}(t)-t \boldsymbol{\mu})_{I}>\boldsymbol{x}_{I} \\
\exists_{t \in[0, T]} & \sqrt{c_{j ; u}} Y_{K}+\frac{1}{\sqrt{u}} \boldsymbol{Z}_{K}\left(t, \boldsymbol{x}_{I}\right)>\frac{j T}{\sqrt{u}}\left(\boldsymbol{\mu}_{K}-\Sigma_{K I} \Sigma_{I I}^{-1} \boldsymbol{\mu}_{I}\right) \\
\sqrt{c_{j ; u}} Y_{J}+\frac{1}{\sqrt{u}} \boldsymbol{Z}_{J}\left(t, \boldsymbol{x}_{I}\right)>\sqrt{u}\left(\boldsymbol{b}_{J}-\Sigma_{J I} \Sigma_{I I}^{-1} \boldsymbol{b}_{I}+\left(\boldsymbol{\mu}_{J}-\Sigma_{J I} \Sigma_{I I}^{-1} \boldsymbol{\mu}_{I}\right) \frac{j T}{u}\right)
\end{array}\right\} .
\end{aligned}
$$

and

$$
\begin{gathered}
R_{T}(u)=\exp \left(\frac{u g_{I}\left(t_{0}\right)}{2}\right) \frac{T}{\sqrt{u}} \sum_{-N_{u}-1 \leq j \leq N_{u}} \frac{1}{c_{j ; u}^{m / 2}} \exp \left(-\frac{1}{2} u g_{I}\left(t_{0}+\frac{j T}{u}\right)\right) \\
\times \int_{\mathbb{R}^{m}} f_{j ; u}\left(T, \boldsymbol{x}_{I}\right) P_{j ; u}\left(T, \boldsymbol{x}_{I}\right) d \boldsymbol{x}_{I} .
\end{gathered}
$$

We shall prove in Section 5.4 that

$$
\lim _{u \rightarrow \infty} R_{T}(u)=t_{0}^{-m / 2} \mathcal{H}_{I}(T) \int_{-\infty}^{\infty} e^{-\frac{g_{I}^{\prime \prime}\left(t_{0}\right) x^{2}}{4}} \psi(x) d x
$$

implying thus (34) (recall that $\mathcal{H}_{I}(T)<\infty$ by Lemma 4.2 ).

We shall conclude this section with a result which is needed to prove the sub-additivity property of $\mathcal{H}(T), T>0$. In the following for any fixed $S \in \mathbb{R}, T>0$ we set

$$
\triangle_{u}(S, T)=\left[t_{0}+S u^{-1}, t_{0}+(S+T) u^{-1}\right] .
$$


Note in passing that if $S=T$, then $\triangle_{u}(S, T)=\triangle_{1 ; u}$.

Lemma 4.4. For any fixed $S \in \mathbb{R}, T>0$, we have as $u \rightarrow \infty$

$$
\mathbb{P}\left\{\exists_{t \in \triangle_{u}(S, T)}(\boldsymbol{X}(t))_{I}>\sqrt{u}\left(\boldsymbol{\alpha}_{I}+t \boldsymbol{\mu}_{I}\right)\right\} \sim \frac{\mathbb{P}\left\{\boldsymbol{Y}_{K}>\mathbf{0}_{K}\right\}}{\sqrt{\left(2 \pi t_{0}\right)^{m}\left|\Sigma_{I I}\right|}} \mathcal{H}_{I}(T) u^{-\frac{m}{2}} e^{-\frac{u}{2} g_{I}\left(t_{0}\right)} .
$$

Proof: As in (36) for all $u>0$ we have

$$
\begin{aligned}
& \mathbb{P}\left\{\exists_{t \in \triangle_{u}(S, T)}(\boldsymbol{X}(t))_{I}>\sqrt{u}\left(\boldsymbol{\alpha}_{I}+t \boldsymbol{\mu}_{I}\right)\right\} \\
& =\frac{u^{-m / 2}}{\sqrt{(2 \pi)^{m}\left|\Sigma_{I I}\right|}} \frac{1}{\left(c_{u}(S)\right)^{m / 2}} \exp \left(-\frac{1}{2} u g_{I}\left(t_{0}+\frac{S}{u}\right)\right) \int_{\mathbb{R}^{m}} f_{u}\left(S, \boldsymbol{x}_{I}\right) P_{u}\left(S, T, \boldsymbol{x}_{I}\right) d \boldsymbol{x}_{I},
\end{aligned}
$$

where $c_{u}(S)=t_{0}+S / u$, and with $\boldsymbol{b}_{u}(S)=\boldsymbol{b}+\boldsymbol{\mu} S / u$

$$
\begin{aligned}
& f_{u}\left(S, \boldsymbol{x}_{I}\right)=\exp \left(\frac{1}{c_{u}(S)} \boldsymbol{x}_{I}^{\top} \Sigma_{I I}^{-1}\left(\boldsymbol{b}_{u}(S)\right)_{I}-\frac{1}{2 u c_{u}(S)} \boldsymbol{x}_{I}^{\top} \Sigma_{I I}^{-1} \boldsymbol{x}_{I}\right), \\
& P_{u}\left(S, T, \boldsymbol{x}_{I}\right)=\mathbb{P}\left\{\begin{array}{cl} 
& (\boldsymbol{X}(t)-t \boldsymbol{\mu})_{I}>\boldsymbol{x}_{I} \\
\exists_{t \in[0, T]} & \sqrt{c_{u}(S)} Y_{K}+\frac{1}{\sqrt{u}} \boldsymbol{Z}_{K}\left(t, \boldsymbol{x}_{I}\right)>\frac{S}{\sqrt{u}}\left(\boldsymbol{\mu}_{K}-\Sigma_{K I} \Sigma_{I I}^{-1} \boldsymbol{\mu}_{I}\right) \\
& \sqrt{c_{u}(S)} Y_{J}+\frac{1}{\sqrt{u}} \boldsymbol{Z}_{J}\left(t, \boldsymbol{x}_{I}\right)>\sqrt{u}\left(\boldsymbol{b}_{J}-\Sigma_{J I} \Sigma_{I I}^{-1} \boldsymbol{b}_{I}+\left(\boldsymbol{\mu}_{J}-\Sigma_{J I} \Sigma_{I I}^{-1} \boldsymbol{\mu}_{I}\right) \frac{S}{u}\right)
\end{array}\right\} .
\end{aligned}
$$

We adopt the same notation introduced in (66) and (67). Next, we have the following upper bounds:

$$
f_{u}\left(S, \boldsymbol{x}_{I}\right) \leq e^{\frac{\boldsymbol{x}_{I}^{\top}\left(\Sigma_{I I}^{-1} \boldsymbol{b}_{I}+\boldsymbol{\varepsilon}_{I}^{\boldsymbol{x}_{I}}\right)}{t_{0}+\varepsilon\left(\boldsymbol{x}_{I}\right)}}, \quad P_{u}\left(S, T, \boldsymbol{x}_{I}\right) \leq \mathbb{P}\left\{\exists_{t \in[0, T]}(\boldsymbol{X}(t)-t \boldsymbol{\mu})_{I}>\boldsymbol{x}_{I}\right\}
$$

Furthermore, by (33)

$$
\int_{\mathbb{R}^{m}} e^{\frac{\boldsymbol{x}_{I}^{\top}\left(\Sigma_{I I}^{-1} \boldsymbol{b}_{I}+\boldsymbol{\varepsilon}_{I}^{\left.\boldsymbol{x}_{I}\right)}\right.}{t_{0}+\varepsilon\left(\boldsymbol{x}_{I}\right)}} \mathbb{P}\left\{\exists_{t \in[0, T]}(\boldsymbol{X}(t)-t \boldsymbol{\mu})_{I}>\boldsymbol{x}_{I}\right\} d \boldsymbol{x}_{I}<\infty .
$$

Consequently, the claim follows from the dominated convergence theorem by letting $u \rightarrow \infty$, and thus the proof is complete.

Finiteness and positivity of $\mathcal{H}_{I}$. Recall that $I$ with $m=\sharp I$ elements is the essential index set of the quadratic programming problem $P_{\Sigma}(\boldsymbol{b})$ where

$$
\boldsymbol{b}=\boldsymbol{b}\left(t_{0}\right)=\boldsymbol{\alpha}+\boldsymbol{\mu} t_{0}
$$

We first prove the sub-additivity of $\mathcal{H}_{I}(T), T>0$.

Lemma 4.5. For any $S, T$ positive we have $\mathcal{H}_{I}(S+T) \leq \mathcal{H}_{I}(S)+\mathcal{H}_{I}(T)$. Moreover,

$$
\mathcal{H}_{I}=\inf _{T>0} \frac{1}{T} \mathcal{H}_{I}(T)<\infty
$$

Proof: Note that

$$
\begin{aligned}
\mathbb{P}\left\{\exists_{t \in\left[t_{0}, t_{0}+(S+T) u^{-1}\right]}(\boldsymbol{X}(t))_{I}>\sqrt{u}\left(\boldsymbol{\alpha}_{I}+t \boldsymbol{\mu}_{I}\right)\right\} \leq & \mathbb{P}\left\{\exists_{t \in\left[t_{0}, t_{0}+S u^{-1}\right]}(\boldsymbol{X}(t))_{I}>\sqrt{u}\left(\boldsymbol{\alpha}_{I}+t \boldsymbol{\mu}_{I}\right)\right\} \\
& +\mathbb{P}\left\{\exists_{t \in\left[t_{0}+S u^{-1}, t_{0}+(S+T) u^{-1}\right]}(\boldsymbol{X}(t))_{I}>\sqrt{u}\left(\boldsymbol{\alpha}_{I}+t \boldsymbol{\mu}_{I}\right)\right\}
\end{aligned}
$$

Using the result of Lemma 4.4 the proof of the sub-additivity follows. The second claim follows directly from Fekete's lemma. This completes the proof.

Lemma 4.6. For any $t>0$

$$
\int_{\mathbb{R}^{m}} e^{\frac{\boldsymbol{x}_{I}^{\top} \Sigma_{I I}^{-1} \boldsymbol{b}_{I}}{t_{0}}} \mathbb{P}\left\{(\boldsymbol{X}(t)-\boldsymbol{\mu} t)_{I}>\boldsymbol{x}_{I}\right\} d \boldsymbol{x}_{I}=\frac{t_{0}^{m}}{\prod_{i \in I}\left(\Sigma_{I I}^{-1} \boldsymbol{b}_{I}\right)_{i}}>0 .
$$


Proof: First note that the solution of the quadratic programming problem $P_{\Sigma}(\boldsymbol{b})$ is such that

$$
\prod_{i \in I}\left(\Sigma_{I I}^{-1} \boldsymbol{b}_{I}\right)_{i}>0
$$

Since

$$
\mathbb{E}\left\{e^{\boldsymbol{s}_{I}^{\top}(\boldsymbol{X}(t))_{I}}\right\}=e^{t \boldsymbol{s}_{I}^{\top} \Sigma_{I I} \boldsymbol{s}_{I} / 2}, \quad s \in \mathbb{R}^{d}, t>0
$$

for any $a>0$ we have

$$
\begin{aligned}
\int_{\mathbb{R}^{m}} e^{a \boldsymbol{x}_{I}^{\top} \Sigma_{I I}^{-1} \boldsymbol{b}_{I}} \mathbb{P}\left\{(\boldsymbol{X}(t)-\boldsymbol{\mu} t)_{I}>\boldsymbol{x}_{I}\right\} d \boldsymbol{x}_{I} & =e^{-a t \boldsymbol{\mu}_{I}^{\top} \Sigma_{I I}^{-1} \boldsymbol{b}_{I}} \int_{\mathbb{R}^{m}} e^{a \boldsymbol{y}_{I}^{\top} \Sigma_{I I}^{-1} \boldsymbol{b}_{I}}\left(\int_{\boldsymbol{z}_{I} \geq \boldsymbol{y}_{I}} \phi_{t \Sigma_{I I}}\left(\boldsymbol{z}_{I}\right) d \boldsymbol{z}_{I}\right) d \boldsymbol{y}_{I} \\
& =\frac{a^{-m}}{\prod_{i \in I}\left(\Sigma_{I I}^{-1} \boldsymbol{b}_{I}\right)_{i}} e^{-a \boldsymbol{\mu}_{I}^{\top} \Sigma_{I I}^{-1} \boldsymbol{b}_{I}} \int_{\mathbb{R}^{m}} e^{a \boldsymbol{z}_{I}^{\top} \Sigma_{I I}^{-1} \boldsymbol{b}_{I} \phi_{t \Sigma_{I I}}\left(\boldsymbol{z}_{I}\right) d \boldsymbol{z}_{I}} \\
& =\frac{a^{-m}}{\prod_{i \in I}\left(\Sigma_{I I}^{-1} \boldsymbol{b}_{I}\right)_{i}} e^{-a t \boldsymbol{\mu}_{I}^{\top} \Sigma_{I I}^{-1} \boldsymbol{b}_{I}+a^{2} t \frac{\boldsymbol{b}_{I}^{\top} \Sigma_{I I}^{-1} \boldsymbol{b}_{I}}{2}}
\end{aligned}
$$

where

$$
\phi_{t \Sigma_{I I}}\left(\boldsymbol{z}_{I}\right)=\frac{1}{\sqrt{(2 \pi t)^{m}\left|\Sigma_{I I}\right|}} \exp \left(-\frac{1}{2 t} \boldsymbol{z}_{I}^{\top} \Sigma_{I I}^{-1} \boldsymbol{z}_{I}\right) .
$$

In view of (16) we have that $g_{I}^{\prime}\left(t_{0}\right)=0$, (recall that $\left.\boldsymbol{b}=\boldsymbol{\alpha}+\boldsymbol{\mu} t_{0}\right)$ hence

$$
-\frac{\boldsymbol{\mu}_{I}^{\top} \Sigma_{I I}^{-1} \boldsymbol{b}_{I}}{t_{0}}+\frac{\boldsymbol{b}_{I}^{\top} \Sigma_{I I}^{-1} \boldsymbol{b}_{I}}{2 t_{0}^{2}}=0
$$

implying thus $\boldsymbol{\mu}_{I}^{\top} \Sigma_{I I}^{-1} \boldsymbol{b}_{I}>0$. Moreover, choosing $a=1 / t_{0}$, where

$$
t_{0}=\sqrt{\frac{\boldsymbol{\alpha}_{I}^{\top} \Sigma_{I I}^{-1} \boldsymbol{\alpha}_{I}}{\boldsymbol{\mu}_{I}^{\top} \Sigma_{I I}^{-1} \boldsymbol{\mu}_{I}}}>0
$$

establishes the claim.

Lemma 4.7. We have

$$
\mathcal{H}_{I} \geq \frac{t_{0}^{m-1} \boldsymbol{\mu}_{I}^{\top} \Sigma_{I I}^{-1} \boldsymbol{b}_{I}}{16 \prod_{i \in I}\left(\Sigma_{I I}^{-1} \boldsymbol{b}_{I}\right)_{i}}>0
$$

Proof: Suppose that $\delta>0$ and let $n$ be any integer. Application of Bonferroni's inequality yields

$$
\begin{aligned}
\mathcal{H}_{I}(\delta n) \geq & \int_{\mathbb{R}^{m}} e^{\frac{\boldsymbol{x}_{I}^{\top} \Sigma_{I I}^{-1} \boldsymbol{b}_{I}}{t_{0}}} \mathbb{P}\left\{\exists k \in\{1, \ldots, n\}:(\boldsymbol{X}(\delta k)-\boldsymbol{\mu}(\delta k))_{I}>\boldsymbol{x}_{I}\right\} d \boldsymbol{x}_{I} \\
\geq & \int_{\mathbb{R}^{m}} e^{\frac{\boldsymbol{x}_{I}^{\top} \Sigma_{I I}^{-1} \boldsymbol{b}_{I}}{t_{0}}} \sum_{k=1}^{n} \mathbb{P}\left\{(\boldsymbol{X}(\delta k)-\boldsymbol{\mu}(\delta k))_{I}>\boldsymbol{x}_{I}\right\} d \boldsymbol{x}_{I} \\
& -\int_{\mathbb{R}^{m}} e^{\frac{\boldsymbol{x}_{I}^{\top} \Sigma_{I I}^{-1} \boldsymbol{b}_{I}}{t_{0}}} \sum_{k=1}^{n-1} \sum_{l=k+1}^{n} \mathbb{P}\left\{(\boldsymbol{X}(\delta k)-\boldsymbol{\mu}(\delta k))_{I}>\boldsymbol{x}_{I},(\boldsymbol{X}(\delta l)-\boldsymbol{\mu}(\delta l))_{I}>\boldsymbol{x}_{I}\right\} d \boldsymbol{x}_{I} \\
=: & \mathcal{I}_{1}-\mathcal{I}_{2} .
\end{aligned}
$$

By Lemma 4.6 we have

Next, since

$$
\mathcal{I}_{1}=n Q, \quad Q:=\frac{t_{0}^{m}}{\prod_{i=1}^{m}\left(\Sigma_{I I}^{-1} \boldsymbol{b}_{I}\right)_{i}} .
$$

$$
\mathbb{P}\left\{(\boldsymbol{X}(\delta k)-\boldsymbol{\mu}(\delta k))_{I}>\boldsymbol{x}_{I},(\boldsymbol{X}(\delta l)-\boldsymbol{\mu}(\delta l))_{I}>\boldsymbol{x}_{I}\right\} \leq \mathbb{P}\left\{\frac{1}{2}(\boldsymbol{X}(\delta k)+\boldsymbol{X}(\delta l)-\boldsymbol{\mu}(\delta k+\delta l))_{I}>\boldsymbol{x}_{I}\right\}
$$

by Lemma 4.6

$$
\mathcal{I}_{2} \leq \sum_{k=1}^{n-1} \sum_{l=k+1}^{n} \int_{\mathbb{R}^{m}} e^{\frac{\boldsymbol{x}_{I}^{\top} \Sigma_{I I}^{-1} \boldsymbol{b}_{I}}{t_{0}}} \mathbb{P}\left\{\frac{1}{2}(\boldsymbol{X}(\delta k)+\boldsymbol{X}(\delta l)-\boldsymbol{\mu}(\delta k+\delta l))_{I}>\boldsymbol{x}_{I}\right\} d \boldsymbol{x}_{I}
$$




$$
\begin{aligned}
& =\sum_{k=1}^{n-1} \sum_{l=k+1}^{n} \int_{\mathbb{R}^{m}} e^{\frac{\boldsymbol{x}_{I}^{\top} \Sigma_{I I}^{-1} b_{I}}{t_{0}}} \mathbb{P}\left\{\sqrt{\frac{3 \delta k+\delta l}{4}}(\boldsymbol{X}(1))_{I}-\boldsymbol{\mu}_{I} \frac{3 \delta k+\delta l}{4}>\boldsymbol{x}_{I}+\frac{\boldsymbol{\mu}_{I} \delta(l-k)}{4}\right\} d \boldsymbol{x}_{I} \\
& =Q \sum_{k=1}^{n-1} \sum_{l=k+1}^{n} e^{-\frac{\boldsymbol{\mu}_{I}^{\top} \Sigma_{I I}^{-1} \boldsymbol{b}_{I} \delta}{4 t_{I}}(l-k)} .
\end{aligned}
$$

Since by (42) we have $\boldsymbol{\mu}_{I}^{\top} \Sigma_{I I}^{-1} \boldsymbol{b}_{I}>0$, hence

$$
\mathcal{I}_{2} \leq Q n \int_{0}^{\infty} e^{-\frac{\boldsymbol{\mu}_{I}^{\top} \Sigma_{I I}^{-1} \boldsymbol{b}_{I} \delta}{4 t_{0}} x} d x=Q n \frac{4 t_{0}}{\delta \boldsymbol{\mu}_{I}^{\top} \Sigma_{I I}^{-1} \boldsymbol{b}_{I}} .
$$

By Lemma 4.5

$$
\mathcal{H}_{I}=\inf _{T>0} \frac{1}{T} \mathcal{H}_{I}(T) \geq \inf _{n>0} \frac{\mathcal{I}_{1}-\mathcal{I}_{2}}{\delta n}=\frac{Q}{\delta}\left(1-\frac{1}{\delta} \frac{4 t_{0}}{\boldsymbol{\mu}_{I}^{\top} \Sigma_{I I}^{-1} \boldsymbol{b}_{I}}\right) .
$$

Since $\delta>0$ was arbitrary, as in [20],

$$
\mathcal{H}_{I} \geq \max _{\delta>0} \frac{Q}{\delta}\left(1-\frac{1}{\delta} \frac{4 t_{0}}{\boldsymbol{\mu}_{I}^{\top} \Sigma_{I I}^{-1} \boldsymbol{b}_{I}}\right) \geq Q \frac{\boldsymbol{\mu}_{I}^{\top} \Sigma_{I I}^{-1} \boldsymbol{b}_{I}}{16 t_{0}}>0,
$$

establishing the proof.

Estimation of double-sum. In this subsection we shall show that as $u \rightarrow \infty$ and then $T \rightarrow \infty$

$$
\Pi(u)=o\left(p_{1}(u)\right) .
$$

First, note that

$$
\begin{aligned}
p_{i, j ; u} & =\mathbb{P}\left\{\exists_{s \in \Delta_{i ; u}} \boldsymbol{X}(s)>\sqrt{u}(\boldsymbol{\alpha}+s \boldsymbol{\mu}), \exists_{t \in \Delta_{j ; u}} \boldsymbol{X}(\boldsymbol{t})>\sqrt{u}(\boldsymbol{\alpha}+t \boldsymbol{\mu})\right\} \\
& \leq \mathbb{P}\left\{\exists_{(s, t) \in \Delta_{i ; u} \times \Delta_{j ; u}}(\boldsymbol{X}(s)+\boldsymbol{X}(t))_{I}>\sqrt{u}\left(2 \boldsymbol{\alpha}_{I}+(s+t) \boldsymbol{\mu}_{I}\right)\right\} \\
& =\mathbb{P}\left\{\exists_{(s, t) \in[0, T]^{2}} \frac{1}{2}\left(\boldsymbol{X}\left(t_{0}+\frac{i T+s}{u}\right)+\boldsymbol{X}\left(t_{0}+\frac{j T+t}{u}\right)\right)_{I}>\sqrt{u}\left(\boldsymbol{\alpha}_{I}+\left(t_{0}+\frac{(i+j) T+s+t}{2 u}\right) \boldsymbol{\mu}_{I}\right)\right\} .
\end{aligned}
$$

Next we rewrite for $(s, t) \in[0, T]^{2}$

$$
\begin{aligned}
\boldsymbol{X}\left(t_{0}+\right. & \left.\frac{i T+s}{u}\right)+\boldsymbol{X}\left(t_{0}+\frac{j T+t}{u}\right) \\
= & \left\{2 \boldsymbol{X}\left(t_{0}+\frac{i T}{u}\right)\right\}+\left\{\left(\boldsymbol{X}\left(t_{0}+\frac{i T+s}{u}\right)-\boldsymbol{X}\left(t_{0}+\frac{i T}{u}\right)\right)+\left(\boldsymbol{X}\left(t_{0}+\frac{(i+1) T}{u}\right)-\boldsymbol{X}\left(t_{0}+\frac{i T}{u}\right)\right)\right\} \\
& \quad+\left\{\boldsymbol{X}\left(t_{0}+\frac{j T}{u}\right)-\boldsymbol{X}\left(t_{0}+\frac{(i+1) T}{u}\right)\right\}+\left\{\boldsymbol{X}\left(t_{0}+\frac{j T+t}{u}\right)-\boldsymbol{X}\left(t_{0}+\frac{j T}{u}\right)\right\} .
\end{aligned}
$$

Note that all the processes (or random variables) inside consecutive $\{\ldots\}$ are mutually independent. Consequently,

$$
\begin{aligned}
& \frac{1}{2}\left(\boldsymbol{X}\left(t_{0}+\frac{i T+s}{u}\right)+\boldsymbol{X}\left(t_{0}+\frac{j T+t}{u}\right)\right)_{I} \\
& \quad \stackrel{d}{=}\left(\boldsymbol{Z}_{i, j ; u}\right)_{I}+\frac{1}{2 \sqrt{u}}\left(\boldsymbol{X}_{1}(s)+\boldsymbol{X}_{1}(T)+\boldsymbol{X}_{2}(t)\right)_{I}, \quad(s, t) \in[0, T]^{2},
\end{aligned}
$$

where $\boldsymbol{X}_{1}$ and $\boldsymbol{X}_{2}$ are independent copies of $\boldsymbol{X}$, which are also independent of $\boldsymbol{Z}_{i, j ; u} \stackrel{d}{=} \sqrt{c_{i, j ; u}} A \boldsymbol{N}$, with covariance matrix $\Sigma_{i, j ; u}=c_{i, j ; u} \Sigma$, where $c_{i, j ; u}=t_{0}+\frac{(j+3 i-1) T}{4 u}$ and $\boldsymbol{N}=\left(N_{1}, \ldots, N_{d}\right)$ has independent $\mathcal{N}(0,1)$ components. Next, set

$$
\boldsymbol{b}_{i, j ; u}=\boldsymbol{b}\left(t_{0}+\frac{(i+j) T}{2 u}\right)
$$

It follows from (44) and (45) that

$$
\begin{aligned}
\mathbb{P} & \left\{\exists_{(s, t) \in \Delta_{i, u} \times \Delta_{j, u}}(\boldsymbol{X}(s)+\boldsymbol{X}(t))_{I}>\sqrt{u}\left(2 \boldsymbol{\alpha}_{I}+(s+t) \boldsymbol{\mu}_{I}\right)\right\} \\
& =\mathbb{P}\left\{\exists_{(s, t) \in[0, T]^{2}}\left(\boldsymbol{Z}_{i, j ; u}\right)_{I}+\frac{1}{2 \sqrt{u}}\left(\boldsymbol{X}_{1}(s)+\boldsymbol{X}_{1}(T)+\boldsymbol{X}_{2}(t)-(s+t) \boldsymbol{\mu}\right)_{I}>\sqrt{u}\left(\boldsymbol{b}_{i, j ; u}\right)_{I}\right\}
\end{aligned}
$$




$$
\begin{aligned}
= & \frac{u^{-m / 2}}{\sqrt{(2 \pi)^{m}\left|\left(\sum_{i, j ; u}\right)_{I I}\right|}} \int_{\mathbb{R}^{m}} \exp \left(-\frac{1}{2}\left(\sqrt{u}\left(\boldsymbol{b}_{i, j ; u}\right)_{I}-\frac{\boldsymbol{x}_{I}}{\sqrt{u}}\right)^{\top}\left(\sum_{i, j ; u}\right)_{I I}^{-1}\left(\sqrt{u}\left(\boldsymbol{b}_{i, j ; u}\right)_{I}-\frac{\boldsymbol{x}_{I}}{\sqrt{u}}\right)\right) \\
& \times \mathbb{P}\left\{\exists_{(s, t) \in[0, T]^{2}} \frac{1}{2}\left(\boldsymbol{X}_{1}(s)+\boldsymbol{X}_{1}(T)+\boldsymbol{X}_{2}(t)-(s+t) \boldsymbol{\mu}\right)_{I}>\boldsymbol{x}_{I}\right\} d \boldsymbol{x}_{I} .
\end{aligned}
$$

In particular for $i=0, j=2$ using (46) and similar arguments as in the proof of Lemma 4.4 we have

$$
\begin{gathered}
\mathbb{P}\left\{\exists_{(s, t) \in \Delta_{0, u} \times \Delta_{2, u}}(\boldsymbol{X}(s)+\boldsymbol{X}(t))_{I}>\sqrt{u}\left(2 \boldsymbol{\alpha}_{I}+\boldsymbol{\mu}_{I}(s+t)\right)\right\} \\
\sim \widetilde{\mathcal{H}_{I}}(T) \frac{u^{-m / 2}}{\sqrt{\left(2 \pi t_{0}\right)^{m}\left|\Sigma_{I I}\right|}} \exp \left(-\frac{u}{2} g_{I}\left(t_{0}\right)\right) \exp (-3 a T),
\end{gathered}
$$

where $a=\frac{g_{I}\left(t_{0}\right)}{8 t_{0}}$ and

$$
\widetilde{\mathcal{H}_{I}}(T)=\int_{\mathbb{R}^{m}} e^{\frac{\boldsymbol{x}_{I}^{\top} \Sigma_{I I}^{-1} \boldsymbol{b}_{I}}{t_{0}}} \mathbb{P}\left\{\exists_{(s, t) \in[0, T]^{2}} \frac{1}{2}\left(\boldsymbol{X}_{1}(s)+\boldsymbol{X}_{1}(T)+\boldsymbol{X}_{2}(t)-\boldsymbol{\mu}(s+t)\right)_{I}>\boldsymbol{x}_{I}\right\} d \boldsymbol{x}_{I} .
$$

Note that in a similar vein as in Lemma 4.2 we can prove the finiteness of $\widetilde{\mathcal{H}_{I}}(T)$.

We shall need an upper bound for $p_{i, j ; u}$ derived in the following lemma.

Lemma 4.8. For any fixed $T>0$, there exists some small $\varepsilon>0$ such that, for all $i, j$ satisfying $-N_{u} \leq i<j \leq N_{u}$,

$$
\begin{aligned}
p_{i, j ; u} \leq & C T^{2} e^{2 a T} u^{-m / 2} \exp \left(-\frac{g_{I}\left(t_{0}\right)}{2} u\right) \\
& \times \exp \left(-\frac{g_{I}^{\prime \prime}\left(t_{0}\right)-\varepsilon}{4}\left(\frac{i T}{\sqrt{u}}\right)^{2}\right) \exp \left(-a_{\varepsilon}((j-i+1) T)\right)
\end{aligned}
$$

holds for some constant $C>0$ independent of $i, j, u$ and $T$, when $u$ is large, where

$$
a_{\varepsilon}=\frac{1}{2}\left(\frac{g_{I}\left(t_{0}\right)-\varepsilon}{4 t_{0}+\varepsilon}-\frac{\varepsilon\left(g_{I}^{\prime \prime}\left(t_{0}\right)-\varepsilon\right)}{2}\right), \quad a=a_{0}=\frac{g_{I}\left(t_{0}\right)}{8 t_{0}} .
$$

Proof: . In view of (44) and (46) we have (recall $\left.\boldsymbol{b}_{i, j ; u}=\boldsymbol{b}\left(t_{0}+\frac{(i+j) T}{2 u}\right)\right)$

$$
\begin{aligned}
p_{i, j ; u} \leq & \frac{u^{-m / 2}}{\sqrt{(2 \pi)^{m}\left|\left(\Sigma_{i, j ; u}\right)_{I I}\right|}} \int_{\mathbb{R}^{m}} \exp \left(-\frac{1}{2}\left(\sqrt{u}\left(\boldsymbol{b}_{i, j ; u}\right)_{I}-\frac{\boldsymbol{x}_{I}}{\sqrt{u}}\right)^{\top}\left(\Sigma_{i, j ; u}\right)_{I I}^{-1}\left(\sqrt{u}\left(\boldsymbol{b}_{i, j ; u}\right)_{I}-\frac{\boldsymbol{x}_{I}}{\sqrt{u}}\right)\right) \\
& \times \mathbb{P}\left\{\exists_{(s, t) \in[0, T]^{2}} \frac{1}{2}\left(\boldsymbol{X}_{1}(s)+\boldsymbol{X}_{1}(T)+\boldsymbol{X}_{2}(t)-\boldsymbol{\mu}(s+t)\right)_{I}>\boldsymbol{x}_{I}\right\} d \boldsymbol{x}_{I} .
\end{aligned}
$$

Let for $T$ positive

$$
\widetilde{\mathcal{H}}_{I, i, j ; u}(T)=\int_{\mathbb{R}^{m}} e^{\frac{\boldsymbol{x}_{I}^{\top} \Sigma_{I I}^{-1} \boldsymbol{b}\left(t_{0}+\frac{(i+j) T}{2 u}\right)_{I}}{t_{0}+\frac{j+3 i-1}{4 u}}} \mathbb{P}\left\{\exists_{(s, t) \in[0, T]^{2}} \frac{1}{2}\left(\boldsymbol{X}_{1}(s)+\boldsymbol{X}_{1}(T)+\boldsymbol{X}_{2}(t)-\boldsymbol{\mu}(s+t)\right)_{I}>\boldsymbol{x}_{I}\right\} d \boldsymbol{x}_{I} .
$$

Since $\widetilde{\mathcal{H}}_{I, i, j ; u}(T) \rightarrow \widetilde{\mathcal{H}_{I}}(T)$ as $u \rightarrow \infty$ uniformly with respect to $-N_{u} \leq i<j \leq N_{u}$ we have that for large $u$

$$
\widetilde{\mathcal{H}}_{I, i, j ; u}(T) \leq \text { const } \widetilde{\mathcal{H}}_{I}(T)
$$

Now for the expression in the exponent in (49) we have that

$$
\begin{aligned}
& \left(\sqrt{u}\left(\boldsymbol{b}_{i, j ; u}\right)_{I}-\frac{\boldsymbol{x}_{I}}{\sqrt{u}}\right)^{\top}\left(\Sigma_{i, j ; u}\right)_{I I}^{-1}\left(\sqrt{u}\left(\boldsymbol{b}_{i, j ; u}\right)_{I}-\frac{\boldsymbol{x}_{I}}{\sqrt{u}}\right) \\
& =u \frac{1}{c_{i, j ; u}}\left(\boldsymbol{b}_{i, j ; u}\right)_{I}^{\top} \Sigma_{I I}^{-1}\left(\boldsymbol{b}_{i, j ; u}\right)_{I}-2 \frac{1}{c_{i, j ; u}} \boldsymbol{x}_{I}^{\top} \Sigma_{I I}^{-1}\left(\boldsymbol{b}_{i, j ; u}\right)_{I}+\frac{1}{u c_{i, j ; u}} \boldsymbol{x}_{I}^{\top} \Sigma_{I I}^{-1} \boldsymbol{x}_{I} \\
& =u \frac{t_{0}+\frac{(i+j) T}{2 u}}{c_{i, j ; u}} g_{I}\left(t_{0}+\frac{(i+j) T}{2 u}\right)-2 \frac{1}{c_{i, j ; u}} \boldsymbol{x}_{I}^{\top} \Sigma_{I I}^{-1}\left(\boldsymbol{b}_{i, j ; u}\right)_{I}+\frac{1}{u c_{i, j ; u}} \boldsymbol{x}_{I}^{\top} \Sigma_{I I}^{-1} \boldsymbol{x}_{I} .
\end{aligned}
$$


It follows that for all $u>0$ large

$$
p_{i, j ; u} \leq C_{0} \widetilde{\mathcal{H}_{I}}(T) \frac{u^{-m / 2}}{\sqrt{\left(2 \pi\left(t_{0}-\varepsilon\right)\right)^{m}\left|\Sigma_{I I}\right|}} \exp \left(-\frac{u}{2}\left(1+\frac{(j-i+1) T}{\left(4 t_{0}+\frac{(3 i+j-1) T}{u}\right) u}\right) g_{I}\left(t_{0}+\frac{(i+j) T}{2 u}\right)\right)
$$

for some $C_{0}>0$ and some small $\varepsilon>0$. Furthermore, we have that for the small $\varepsilon$

$$
\frac{(3 i+j-1) T}{u}<\varepsilon
$$

and

$$
g_{I}\left(t_{0}+\frac{(i+j) T}{2 u}\right) \geq g_{I}\left(t_{0}\right)+\frac{1}{2}\left(g_{I}^{\prime \prime}\left(t_{0}\right)-\varepsilon\right)\left(\frac{(i+j) T}{2 u}\right)^{2}
$$

for all $-N(u) \leq i<j \leq N(u)$ and $u$ large. Moreover, for any $j>i$

$$
\left(\frac{(i+j) T}{2 u}\right)^{2}=\left(\frac{(j-i) T}{2 u}+\frac{i T}{u}\right)^{2} \geq\left(\frac{i T}{u}\right)^{2}+\frac{(j-i) T(i T)}{u^{2}} \geq\left(\frac{i T}{u}\right)^{2}-\varepsilon \frac{(j-i+1) T}{u}
$$

holds for all $u$ large. Consequently, for any $j>i$

$$
\begin{aligned}
& \exp \left(-\frac{u}{2}\left(1+\frac{(j-i+1) T}{\left(4 t_{0}+\frac{(3 i+j-1) T}{u}\right) u}\right) g\left(t_{0}+\frac{(i+j) T}{2 u}\right)\right) \\
& \quad \leq \exp \left(-\frac{u}{2}\left(1+\frac{(j-i+1) T}{\left(4 t_{0}+\varepsilon\right) u}\right)\left(g_{I}\left(t_{0}\right)+\frac{1}{2}\left(g_{I}^{\prime \prime}\left(t_{0}\right)-\varepsilon\right)\left(\left(\frac{i T}{u}\right)^{2}-\varepsilon \frac{(j-i+1) T}{u}\right)\right)\right) \\
& \quad=\exp \left(-\frac{g_{I}\left(t_{0}\right)}{2} u-\frac{g_{I}^{\prime \prime}\left(t_{0}\right)-\varepsilon}{4}\left(\frac{i T}{\sqrt{u}}\right)^{2}-\frac{(j-i+1) T}{2}\left(\frac{g_{I}\left(t_{0}\right)+\frac{g_{I}^{\prime \prime}\left(t_{0}\right)-\varepsilon}{2}\left(\left(\frac{i T}{u}\right)^{2}-\varepsilon \frac{(j-i+1) T}{u}\right)}{4 t_{0}+\varepsilon}-\frac{\varepsilon\left(g_{I}^{\prime \prime}\left(t_{0}\right)-\varepsilon\right)}{2}\right)\right.
\end{aligned}
$$

With the small given positive $\varepsilon$, for all $-N(u) \leq i<j \leq N(u)$ and all large $u$ we have

$$
g_{I}\left(t_{0}\right)+\frac{g_{I}^{\prime \prime}\left(t_{0}\right)-\varepsilon}{2}\left(\left(\frac{i T}{u}\right)^{2}-\varepsilon \frac{(j-i+1) T}{u}\right) \geq g_{I}\left(t_{0}\right)-\varepsilon
$$

Consequently,

$$
\begin{aligned}
& \exp \left(-\frac{u}{2}\left(1+\frac{(j-i+1) T}{\left(4 t_{0}+\frac{(3 i+j-1) T}{u}\right) u}\right) g\left(t_{0}+\frac{(i+j) T}{2 u}\right)\right) \\
& \quad \leq \exp \left(-\frac{g_{I}\left(t_{0}\right)}{2} u\right) \exp \left(-\frac{g_{I}^{\prime \prime}\left(t_{0}\right)-\varepsilon}{4}\left(\frac{i T}{\sqrt{u}}\right)^{2}\right) \exp \left(-a_{\varepsilon}((j-i+1) T)\right)
\end{aligned}
$$

from which we obtain that

$$
\begin{aligned}
p_{i, j ; u} \leq & C \widetilde{\mathcal{H}_{I}}(T) \frac{u^{-m / 2}}{\sqrt{\left(2 \pi\left(t_{0}-\varepsilon\right)\right)^{m}\left|\Sigma_{I I}\right|}} \exp \left(-\frac{g_{I}\left(t_{0}\right)}{2} u\right) \\
& \times \exp \left(-\frac{g_{I}^{\prime \prime}\left(t_{0}\right)-\varepsilon}{4}\left(\frac{i T}{\sqrt{u}}\right)^{2}\right) \exp \left(-a_{\varepsilon}((j-i+1) T)\right)
\end{aligned}
$$

holds when $u$ is large. Next, in order to complete the proof it is sufficient to show that for any positive integer $T$

$$
\widetilde{\mathcal{H}_{I}}(T) \leq T^{2} e^{2 a T} \widetilde{\mathcal{H}_{I}}(1)
$$

For $T, u$ positive define

$$
\begin{gathered}
E_{u}(T)=\left[t_{0}, t_{0}+T u^{-1}\right] \times\left[t_{0}+2 T u^{-1}, t_{0}+3 T u^{-1}\right] \\
E_{u}(k, l)=\left[t_{0}+k u^{-1}, t_{0}+(k+1) u^{-1}\right] \times\left[t_{0}+(2 T+l) u^{-1}, t_{0}+(2 T+l+1) u^{-1}\right], \\
k, l=0,1, \cdots, T-1 .
\end{gathered}
$$


It follow from (47) that

$$
\lim _{u \rightarrow \infty} \frac{\mathbb{P}\left\{\exists_{(s, t) \in E_{u}(T)}(\boldsymbol{X}(s)+\boldsymbol{X}(t))_{I}>\sqrt{u}\left(2 \boldsymbol{\alpha}_{I}+\boldsymbol{\mu}_{I}(s+t)\right)\right\}}{\frac{u^{-m / 2}}{\sqrt{\left(2 \pi t_{0}\right)^{m}\left|\Sigma_{I I}\right|}} \exp \left(-\frac{u}{2} g_{I}\left(t_{0}\right)\right)}=e^{-3 a T} \widetilde{\mathcal{H}_{I}}(T) .
$$

Similarly, we can show that

$$
\lim _{u \rightarrow \infty} \frac{\mathbb{P}\left\{\exists_{(t, w) \in E_{u}(k, l)}(\boldsymbol{X}(s)+\boldsymbol{X}(t))_{I}>\sqrt{u}\left(2 \boldsymbol{\alpha}_{I}+\boldsymbol{\mu}_{I}(s+t)\right)\right\}}{\frac{u^{-m / 2}}{\sqrt{\left(2 \pi t_{0}\right)^{m}\left|\Sigma_{I I}\right|}} \exp \left(-\frac{u}{2} g_{I}\left(t_{0}\right)\right)}=e^{-a(2 T+l-k+1)} \widetilde{\mathcal{H}_{I}}(1) .
$$

Furthermore, since

$$
E_{u}(T) \subset \cup_{k=0}^{T-1} \cup_{l=0}^{T-1} E_{u}(k, l)
$$

we obtain from the above two equalities that

$$
e^{-3 a T} \widetilde{\mathcal{H}_{I}}(T) \leq \sum_{k=0}^{T-1} \sum_{l=0}^{T-1} e^{-a(2 T+l-k+1)} \widetilde{\mathcal{H}_{I}}(1),
$$

which yields that

$$
\begin{gathered}
\widetilde{\mathcal{H}_{I}}(T) \leq e^{a T} \sum_{k=0}^{T-1} \sum_{l=0}^{T-1} e^{-a(l-k+1)} \widetilde{\mathcal{H}_{I}}(1) \\
\leq e^{2 a T} T^{2} \widetilde{\mathcal{H}_{I}}(1)
\end{gathered}
$$

establishing the proof.

Now, we are ready to show (43). Note that

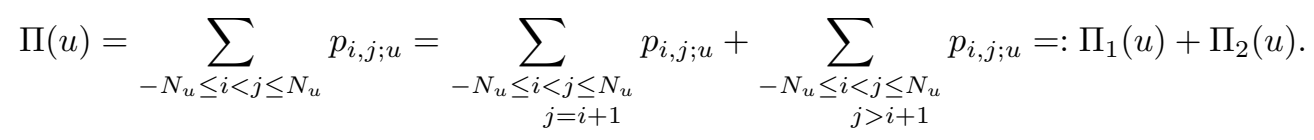

For $\Pi_{1}(u)$ we have

$$
\begin{gathered}
\Pi_{1}(u)=\sum_{i=-N(u)}^{N(u)}\left(\mathbb{P}\left\{\exists_{t \in \triangle_{i ; u}} \boldsymbol{X}(t)>\sqrt{u}(\boldsymbol{\alpha}+\boldsymbol{\mu} t)\right\}+\mathbb{P}\left\{\exists_{t \in \triangle_{(i+1) ; u}} \boldsymbol{X}(t)>\sqrt{u}(\boldsymbol{\alpha}+\boldsymbol{\mu} t)\right\}\right. \\
\left.-\mathbb{P}\left\{\exists_{t \in \triangle_{i ; u} \cup \triangle_{(i+1) ; u}} \boldsymbol{X}(t)>\sqrt{u}(\boldsymbol{\alpha}+\boldsymbol{\mu} t)\right\}\right)=: S_{1}(u)+S_{2}(u)-S_{3}(u) .
\end{gathered}
$$

Recall that we have proved in Lemma 4.5 and Lemma 4.7 that

$$
\lim _{T \rightarrow \infty} T^{-1} \mathcal{H}_{I}(T)=\mathcal{H}_{I} \in(0, \infty),
$$

hence using similar arguments as for (34) to $S_{i}(u), i=1,2,3$, we conclude that

$$
\begin{aligned}
\lim _{T \rightarrow \infty} & \lim _{u \rightarrow \infty} \frac{\Pi_{1}(u)}{u^{(1-m) / 2} \exp \left(-\frac{g_{I}\left(t_{0}\right)}{2} u\right)} \\
& =\frac{1}{\sqrt{\left(2 \pi t_{0}\right)^{m}\left|\Sigma_{I I}\right|}} \int_{-\infty}^{\infty} e^{-\frac{g_{I}^{\prime \prime}\left(t_{0}\right) x^{2}}{4}} \psi(x) d x \lim _{T \rightarrow \infty}\left(\frac{2 \mathcal{H}_{I}(T)}{T}-\frac{\mathcal{H}_{I}(2 T)}{T}\right) \\
& =0 .
\end{aligned}
$$

For $\Pi_{2}(u)$ we have from (48) that, there exists some $\varepsilon>0$, such that

$$
\begin{aligned}
\Pi_{2}(u) \leq & C T e^{2 a T} u^{\frac{1-m}{2}} \exp \left(-\frac{u}{2} g_{I}\left(t_{0}\right)\right) \\
& \times \frac{T}{\sqrt{u}} \sum_{-N_{u} \leq i \leq N_{u}} \exp \left(-\frac{g_{I}^{\prime \prime}\left(t_{0}\right)-\varepsilon}{4}\left(\frac{i T}{\sqrt{u}}\right)^{2}\right) \sum_{j \geq 1} \exp \left(-a_{\varepsilon}(j T)\right) \exp \left(-2 a_{\varepsilon} T\right)
\end{aligned}
$$


holds for all large $u$ with some $C>0$, implying thus

$$
\lim _{T \rightarrow \infty} \lim _{\varepsilon \rightarrow 0} \lim _{u \rightarrow \infty} \frac{\Pi_{2}(u)}{u^{(1-m) / 2} \exp \left(-\frac{g_{I}\left(t_{0}\right)}{2} u\right)}=0,
$$

which establishes (43).

Proof of Theorem 3.1: First note that the finiteness of $\mathcal{H}_{I}$ is established in Lemma 4.5 and the lower bound is obtained in Lemma 4.7. Furthermore, in view of (32), (34), (43) and letting $T \rightarrow \infty$ we obtain (recall (51))

$$
p(u) \sim \frac{1}{\sqrt{\left(2 \pi t_{0}\right)^{m}\left|\Sigma_{I I}\right|}} \mathcal{H}_{I} u^{\frac{1-m}{2}} e^{-\frac{u}{2} g_{I}\left(t_{0}\right)} \int_{\mathbb{R}} e^{-\frac{g_{I}^{\prime \prime}\left(t_{0}\right) x^{2}}{4}} \psi(x) d x, \quad u \rightarrow \infty .
$$

Moreover, by Lemma 4.1

$$
r(u)=o(p(u)), \quad u \rightarrow \infty .
$$

Consequently, the claim follows from (26).

Proof of Theorem 3.3: Define

$$
\widehat{\tau}_{u}=\inf \{t \geq 0: \boldsymbol{X}(t)>(\boldsymbol{\alpha}+\boldsymbol{\mu} t) \sqrt{u}\}
$$

Since $\tau_{u}=u \widehat{\tau}_{u}$, for any $s \in \mathbb{R}$

$$
\begin{aligned}
\mathbb{P}\left\{\frac{\tau_{u}-t_{0} u}{\sqrt{u}} \leq s \mid \tau_{u}<\infty\right\} & =\frac{\mathbb{P}\left\{\frac{\tau_{u}-t_{0} u}{\sqrt{u}} \leq s, \tau_{u}<\infty\right\}}{\mathbb{P}\left\{\tau_{u}<\infty\right\}} \\
& =\frac{\mathbb{P}\left\{u \widehat{\tau}_{u} \leq u t_{0}+\sqrt{u} s\right\}}{P(u)} \\
& =\frac{\mathbb{P}\left\{\exists_{t \in\left[0, t_{0}+s / \sqrt{u}\right]} \boldsymbol{X}(t)>(\boldsymbol{\alpha}+\boldsymbol{\mu} t) \sqrt{u}\right\}}{P(u)} .
\end{aligned}
$$

Using the same arguments as in the proof of Theorem 3.1, we have

$$
\begin{aligned}
\mathbb{P}\left\{\exists_{t \in\left[0, t_{0}+s / \sqrt{u}\right]} \boldsymbol{X}(t)>(\boldsymbol{\alpha}+\boldsymbol{\mu} t) \sqrt{u}\right\} & \sim \mathbb{P}\left\{\exists_{t \in\left[t_{0}-\ln (u) / \sqrt{u}, t_{0}+s / \sqrt{u}\right]} \boldsymbol{X}(t)>(\boldsymbol{\alpha}+\boldsymbol{\mu} t) \sqrt{u}\right\} \\
& \sim \frac{\mathcal{H}_{I}}{\sqrt{\left(2 \pi t_{0}\right)^{m}\left|\Sigma_{I I}\right|}} \int_{-\infty}^{s} e^{-\widetilde{g} \frac{x^{2}}{4}} \psi(x) d x u^{\frac{1-m}{2}} e^{-\frac{\widehat{g}}{2} u}, \quad u \rightarrow \infty .
\end{aligned}
$$

In order to derive the above result the only required modification in the proof of Theorem 3.1 is the replacement of $\sum_{-N_{u}-1 \leq j \leq N_{u}}$ by $\sum_{-N_{u}-1 \leq j \leq\lfloor\sqrt{u} s / T\rfloor}$ in $R_{T}(u)$, see (36). Consequently, the claim follows and thus the proof is complete.

\section{Appendix}

5.1. Quadratic programming problem. This subsection is concerned with discussions on Lemma 2.1, which will be useful for the analysis of the function $g$ in the next subsection. Recall from Lemma 2.1, that $\widetilde{b}$ is the optimal solution of the quadratic programming problem $P_{M}(\boldsymbol{b})$ with the essential index set $I$. Next, we define for $I^{c} \neq \emptyset$

$$
K=\left\{j \in I^{c}: \boldsymbol{b}_{j}=\widetilde{\boldsymbol{b}}_{j}\right\}
$$

We start with some important remarks on Lemma 2.1.

Remark 5.1. i) If there is a unique index set I with maximal number of elements such that (8) holds, then I is the essential index set of $P_{\Sigma}(\boldsymbol{b})$. Otherwise, if there are $I_{1}, \ldots, I_{l}$ index sets which have the same maximal number of elements such that (8) holds, then the unique essential index set say $I=I_{k}$ satisfies additionally (9). 
ii) Note that, for any $I_{1}$ satisfying $\{1, \ldots, d\} \supseteq I_{1} \supset I, \widetilde{\boldsymbol{b}_{I_{1}}}=\widetilde{\boldsymbol{b}}_{I_{1}}$ is the unique solution of the quadratic programming problem $P_{\Sigma_{I_{1} I_{1}}}\left(\boldsymbol{b}_{I_{1}}\right)$. If further

$$
\Sigma_{I_{1} I_{1}}^{-1} \boldsymbol{b}_{I_{1}} \geq \mathbf{0}_{I_{1}}
$$

holds, then $\widetilde{\boldsymbol{b}_{I_{1}}}=\boldsymbol{b}_{I_{1}}$ and

$$
\Sigma_{I_{1} I_{1}}^{-1} \boldsymbol{b}_{I_{1}}=\left(\begin{array}{c}
\Sigma_{I I}^{-1} \boldsymbol{b}_{I} \\
\mathbf{0}_{I_{1} \backslash I}
\end{array}\right),
$$

with $\Sigma_{I I}^{-1} \boldsymbol{b}_{I}>\mathbf{0}_{I}$; see also the proof of 1 of Proposition 2.5 in [19].

iii) Consider the case $d=2$ and let $\boldsymbol{b}$ with $b_{1}=1, b_{2}=b \in(-\infty, 1]$. Suppose for simplicity that $\Sigma$ is a correlation matrix with $\sigma_{12}=\rho \in(-1,1)$. If $b>\rho$, then $\widetilde{\boldsymbol{b}}=\boldsymbol{b}$ and thus $I=\{1,2\}$. If $b=\rho$, then $I=\{1\}, K=\{2\}$. Finally, for $b<\rho$ we have $I=\{1\}, K=\emptyset$.

Lemma 5.2. Let $I \subset\{1, \ldots, d\}$ be the essential index set of the quadratic programming problem $P_{M}(\boldsymbol{b}), \boldsymbol{b} \in \mathbb{R}^{d} \backslash(-\infty, 0]^{d}$. We have, for any $I_{1}$ satisfying $\{1, \ldots, d\} \supseteq I_{1} \supset I$, if $\boldsymbol{b}_{I_{1}}^{\top} M_{I_{1} I_{1}}^{-1} \boldsymbol{b}_{I_{1}}=\boldsymbol{b}_{I}^{\top} M_{I I}^{-1} \boldsymbol{b}_{I}$ holds, then $I_{1} \subseteq I \cup K$, with $K$ given by $(53)$.

Proof: Note that from Remark 5.1 ii) we have $\widetilde{\boldsymbol{b}}_{I_{1}}=\widetilde{\boldsymbol{b}_{I_{1}}}$. In the light of (11),

$$
\boldsymbol{b}_{I_{1}}^{\top} M_{I_{1} I_{1}}^{-1} \widetilde{\boldsymbol{b}}_{I_{1}}=\boldsymbol{b}_{I_{1}}^{\top} M_{I_{1} I_{1}}^{-1} \widetilde{\boldsymbol{b}_{I_{1}}}=\boldsymbol{b}_{I}^{\top} M_{I I}^{-1} \boldsymbol{b}_{I}
$$

and

$$
\widetilde{\boldsymbol{b}}_{I_{1}}^{\top} M_{I_{1} I_{1}}^{-1}\left(\boldsymbol{b}_{I_{1}}-\widetilde{\boldsymbol{b}}_{I_{1}}\right)=\left(\boldsymbol{b}_{I_{1}}-\widetilde{\boldsymbol{b}}_{I_{1}}\right)^{\top} M_{I_{1} I_{1}}^{-1} \widetilde{\boldsymbol{b}_{I_{1}}}=\left(\boldsymbol{b}_{I_{1}}-\widetilde{\boldsymbol{b}}_{I_{1}}\right)_{I}^{\top} M_{I I}^{-1} \boldsymbol{b}_{I}=0 .
$$

Further, since

$$
\begin{aligned}
\boldsymbol{b}_{I_{1}}^{\top} M_{I_{1} I_{1}}^{-1} \boldsymbol{b}_{I_{1}} & =\boldsymbol{b}_{I_{1}}^{\top} M_{I_{1} I_{1}}^{-1}\left(\boldsymbol{b}_{I_{1}}-\widetilde{\boldsymbol{b}}_{I_{1}}\right)+\boldsymbol{b}_{I_{1}}^{\top} M_{I_{1} I_{1}}^{-1} \widetilde{\boldsymbol{b}}_{I_{1}} \\
& =\left(\boldsymbol{b}_{I_{1}}-\widetilde{\boldsymbol{b}}_{I_{1}}\right)^{\top} M_{I_{1} I_{1}}^{-1}\left(\boldsymbol{b}_{I_{1}}-\widetilde{\boldsymbol{b}}_{I_{1}}\right)+\widetilde{\boldsymbol{b}}_{I_{1}}^{\top} M_{I_{1} I_{1}}^{-1}\left(\boldsymbol{b}_{I_{1}}-\widetilde{\boldsymbol{b}}_{I_{1}}\right)+\boldsymbol{b}_{I}^{\top} M_{I I}^{-1} \boldsymbol{b}_{I} \\
& =\left(\boldsymbol{b}_{I_{1}}-\widetilde{\boldsymbol{b}}_{I_{1}}\right)^{\top} M_{I_{1} I_{1}}^{-1}\left(\boldsymbol{b}_{I_{1}}-\widetilde{\boldsymbol{b}}_{I_{1}}\right)+\boldsymbol{b}_{I}^{\top} M_{I I}^{-1} \boldsymbol{b}_{I}
\end{aligned}
$$

we obtain

$$
\left(\boldsymbol{b}_{I_{1}}-\widetilde{\boldsymbol{b}}_{I_{1}}\right)^{\top} M_{I_{1} I_{1}}^{-1}\left(\boldsymbol{b}_{I_{1}}-\widetilde{\boldsymbol{b}}_{I_{1}}\right)=0
$$

thus $\boldsymbol{b}_{I_{1}}=\widetilde{\boldsymbol{b}}_{I_{1}}$, implying that $I_{1} \subseteq I \cup K$ and hence the proof is complete.

5.2. Analysis of $g$. In this subsection we analyze the function

$$
g(t)=\frac{1}{t} \inf _{\boldsymbol{v} \geq \boldsymbol{\alpha}+\boldsymbol{\mu} t} \boldsymbol{v}^{\top} \Sigma^{-1} \boldsymbol{v}
$$

defined already in the Introduction. In the sequel we will denote by $I(t)$ the essential index set of the quadratic programming problem $P_{\Sigma}(\boldsymbol{\alpha}+t \boldsymbol{\mu})$. If $I(t)^{c} \neq \emptyset$ we define

$$
\begin{aligned}
K(t) & =\left\{j \in I(t)^{c}: \Sigma_{j I(t)} \Sigma_{I(t) I(t)}^{-1}(\boldsymbol{\alpha}+\boldsymbol{\mu} t)_{I(t)}=(\boldsymbol{\alpha}+\boldsymbol{\mu} t)_{j}\right\} \\
J(t) & =\left\{j \in I(t)^{c}: \Sigma_{j I(t)} \Sigma_{I(t) I(t)}^{-1}(\boldsymbol{\alpha}+\boldsymbol{\mu} t)_{I(t)}>(\boldsymbol{\alpha}+\boldsymbol{\mu} t)_{j}\right\} .
\end{aligned}
$$

Note that, when analysing the function $g$, the index set $K(t)$ plays the role of $K$ from Section 5.1 .

Lemma 5.3. We have $g \in C(0, \infty)$. 
Proof: Let $h(t)=g(t) t$. For $g \in C(0, \infty)$ it is sufficient that $h \in C(0, \infty)$. In view of Lemma 2.1 we have that for any $t \geq 0$ there exists some $\boldsymbol{v}_{t}^{*}$, so that

$$
h(t)=\left(\boldsymbol{v}_{t}^{*}+\boldsymbol{\alpha}+\boldsymbol{\mu} t\right)^{\top} \Sigma^{-1}\left(\boldsymbol{v}_{t}^{*}+\boldsymbol{\alpha}+\boldsymbol{\mu} t\right),
$$

where

$$
\boldsymbol{v}_{t}^{*}=\left(\begin{array}{c}
\mathbf{0}_{I(t)} \\
\Sigma_{I(t)^{c} I(t)} \Sigma_{I(t) I(t)}^{-1}(\boldsymbol{\alpha}+\boldsymbol{\mu} t)_{I(t)}-(\boldsymbol{\alpha}+\boldsymbol{\mu} t)_{I(t)^{c}}
\end{array}\right) .
$$

For any fixed $t_{1} \in(0, \infty)$, it is easy to see that in a neighbourhood of $t_{1}$, say $\left(t_{1}-\varepsilon, t_{1}+\varepsilon\right)$, with some small $\varepsilon>0$, we have

$$
h(t)=\inf _{\sup _{t \in\left(t_{1}-\varepsilon, t_{1}+\varepsilon\right)} \boldsymbol{v}_{t}^{*} \geq \boldsymbol{v} \geq \mathbf{0}}(\boldsymbol{v}+\boldsymbol{\alpha}+\boldsymbol{\mu} t)^{\top} \Sigma^{-1}(\boldsymbol{v}+\boldsymbol{\alpha}+\boldsymbol{\mu} t), \quad t \in\left(t_{1}-\varepsilon, t_{1}+\varepsilon\right) .
$$

Since for two topological spaces $\mathcal{X}, \mathcal{Y}$ with $\mathcal{Y}$ compact we have

$$
f(x)=\inf _{y \in \mathcal{Y}} q(x, y)
$$

is continuous on $\mathcal{X}$, provided that $q: \mathcal{X} \times \mathcal{Y} \rightarrow \mathbb{R}$ is continuous, we immediately get that $h \in C\left(t_{1}-\varepsilon, t_{1}+\varepsilon\right)$. Consequently, $h \in C(0, \infty)$ follows since $t_{1}$ was chosen arbitrarily.

We show next that

$$
I(t)=\sum_{j} I_{j} \mathbb{I}\left(t \in U_{j}\right),
$$

where $\mathbb{I}(\cdot)$ is the indicator function and $U_{j}$ 's are of the following form

$$
\left(a_{k}, b_{k}\right),\left[a_{k}, b_{k}\right),\left(a_{k}, b_{k}\right],\left[a_{k}, b_{k}\right],\left\{a_{k}\right\},\left(b_{k}, \infty\right),\left[b_{k}, \infty\right)
$$

where $0<a_{k}<b_{k}<\infty$ and $I_{j} \subseteq\{1, \ldots, d\}$. Since point intervals are theoretically possible, we call such function almost piecewise constant set function.

Lemma 5.4. $I(t), t \geq 0$ is an almost piecewise constant set function.

Proof: First, by Lemma 2.1 for any $t \geq 0$ there exists a unique $I(t)$ satisfying

$$
\begin{aligned}
& \Sigma_{I(t) I(t)}^{-1}(\boldsymbol{\alpha}+\boldsymbol{\mu} t)_{I(t)}>\mathbf{0}_{I(t)}, \\
& \Sigma_{I(t)^{c} I(t)} \Sigma_{I(t) I(t)}^{-1}(\boldsymbol{\alpha}+\boldsymbol{\mu} t)_{I(t)} \geq(\boldsymbol{\alpha}+\boldsymbol{\mu} t)_{I(t)^{c}}, \text { if } I(t)^{c} \neq \emptyset .
\end{aligned}
$$

Next, for each $V_{k} \subseteq\{1, \ldots, d\}$ we solve (56) and (57) with $I(t)$ substituted by $V_{k}$ and $I(t)^{c}$ substituted by $V_{k}^{c}=\{1, \ldots, d\} \backslash V_{k}$. Since for each $V_{k}$ the solution is a convex set, by the linearity the solution (if it exists) is in one of the following forms

$$
\left(a_{k}, b_{k}\right),\left[a_{k}, b_{k}\right),\left(a_{k}, b_{k}\right],\left[a_{k}, b_{k}\right],\left\{a_{k}\right\},\left(b_{k}, \infty\right),\left[b_{k}, \infty\right)
$$

Therefore, there exists some finite partition $\left\{U_{1}, \ldots, U_{q}\right\}$ of $[0, \infty)$, with

$$
q \leq \sum_{i=1}^{d}\left(\begin{array}{l}
d \\
i
\end{array}\right)
$$

some constant and $U_{j}$ an interval such that the index set $I(t)=I_{j} \subseteq\{1, \ldots, d\}$ for all $t \in U_{j}^{o}$, hence the proof is complete. 
Lemma 5.5. For the boundary points $t_{j}=\overline{U_{j}} \cap \overline{U_{j+1}}, j=1 \ldots, q-1$, we have $I\left(t_{j}\right) \subset\{1, \ldots, d\}$ and $K\left(t_{j}\right) \neq \emptyset$. Moreover, $I\left(t_{j}\right) \subseteq I(t) \subseteq I\left(t_{j}\right) \cup K\left(t_{j}\right)$ for all $t \in U_{j} \cup U_{j+1}$.

Proof: It follows from Lemma 2.1 that (56) holds for $t=t_{j}$. By continuity, there exists some small $\delta>0$ such that for all $t \in\left(t_{j}-\delta, t_{j}+\delta\right)$

$$
\Sigma_{I\left(t_{j}\right), I\left(t_{j}\right)}^{-1}(\boldsymbol{\alpha}+\boldsymbol{\mu} t)_{I\left(t_{j}\right)}>\mathbf{0}_{I\left(t_{j}\right)} .
$$

This implies that $I\left(t_{j}\right)$ has less than $d$ elements, since otherwise we would have $I(t)=\{1, \ldots, d\}$ for all $t \in \overline{U_{j}} \cap \overline{U_{j+1}}$, a contradiction with the fact that $t_{j}$ is a boundary point. Similarly, if (56) and (57) holds for $t=t_{j}$ with $K\left(t_{j}\right)=\emptyset$, then by continuity we conclude that $I(t)=I\left(t_{j}\right)$ for all $t \in \overline{U_{j}} \cap \overline{U_{j+1}}$, again a contradiction. Thus, $K\left(t_{j}\right) \neq \emptyset$. Now, let $I(t)=I_{j+1}, t \in U_{j+1}^{o}$ and $I(t)=I_{j}, t \in U_{j}^{o}$. Without loss of generality, we only show $I\left(t_{j}\right) \subseteq I_{j}$ since $I\left(t_{j}\right) \subseteq I_{j+1}$ follows with the same arguments. Notice that

$$
\begin{aligned}
& \Sigma_{I\left(t_{j}\right), I\left(t_{j}\right)}^{-1}\left(\boldsymbol{\alpha}+\boldsymbol{\mu} t_{j}\right)_{I\left(t_{j}\right)}>\mathbf{0}_{I\left(t_{j}\right)}, \\
& \Sigma_{K\left(t_{j}\right), I\left(t_{j}\right)} \Sigma_{I\left(t_{j}\right), I\left(t_{j}\right)}^{-1}\left(\boldsymbol{\alpha}+\boldsymbol{\mu} t_{j}\right)_{I\left(t_{j}\right)}=\left(\boldsymbol{\alpha}+\boldsymbol{\mu} t_{j}\right)_{K\left(t_{j}\right)}, \\
& \Sigma_{J\left(t_{j}\right), I\left(t_{j}\right)} \Sigma_{I\left(t_{j}\right), I\left(t_{j}\right)}^{-1}\left(\boldsymbol{\alpha}+\boldsymbol{\mu} t_{j}\right)_{I\left(t_{j}\right)}>\left(\boldsymbol{\alpha}+\boldsymbol{\mu} t_{j}\right)_{J\left(t_{j}\right)} .
\end{aligned}
$$

Since equations in (58) are linear in $t_{j}$ for fixed $I\left(t_{j}\right), K\left(t_{j}\right)$, two cases will be distinguished.

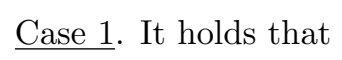

$$
\Sigma_{K\left(t_{j}\right), I\left(t_{j}\right)} \Sigma_{I\left(t_{j}\right), I\left(t_{j}\right)}^{-1}(\boldsymbol{\alpha}+\boldsymbol{\mu} t)_{I\left(t_{j}\right)}>(\boldsymbol{\alpha}+\boldsymbol{\mu} t)_{K\left(t_{j}\right)}
$$

for all $t \in U_{j}^{o}$.

Case 2. There exists some index $i \in K\left(t_{j}\right)$ such that

$$
\Sigma_{i, I\left(t_{j}\right)} \Sigma_{I\left(t_{j}\right), I\left(t_{j}\right)}^{-1}(\boldsymbol{\alpha}+\boldsymbol{\mu} t)_{I\left(t_{j}\right)}<(\boldsymbol{\alpha}+\boldsymbol{\mu} t)_{i}
$$

holds for all $t \in U_{j}^{o}$.

For Case 1 , by continuity we conclude that $I_{j}=I\left(t_{j}\right)$. Next, we focus on Case 2, and show for this case $I\left(t_{j}\right) \subset I_{j}$. Denote $\hat{I}=I\left(t_{j}\right) \cup\{i\}$. We can show that

$$
\Sigma_{\hat{I}, \hat{I}}^{-1}(\boldsymbol{\alpha}+\boldsymbol{\mu} t)_{\hat{I}}>\mathbf{0}_{\hat{I}}
$$

holds for all $t \in U_{j}^{o}$ such that $t-t_{j}$ is small, which, by Remark $5.1 \mathrm{i}$ ), implies that

$$
\sharp I_{j} \geq \sharp \hat{I}=\sharp I\left(t_{j}\right)+1 \text {. }
$$

In fact, denoting $B=\Sigma_{\hat{I}, \hat{I}}^{-1}$ we have

$$
\Sigma_{\hat{I}, \hat{I}}^{-1}(\boldsymbol{\alpha}+\boldsymbol{\mu} t)_{\hat{I}}=\left(\begin{array}{c}
B_{I\left(t_{j}\right) I\left(t_{j}\right)}(\boldsymbol{\alpha}+\boldsymbol{\mu} t)_{I\left(t_{j}\right)}+B_{I\left(t_{j}\right), i}(\boldsymbol{\alpha}+\boldsymbol{\mu} t)_{i} \\
B_{i, I\left(t_{j}\right)}(\boldsymbol{\alpha}+\boldsymbol{\mu} t)_{I\left(t_{j}\right)}+B_{i, i}(\boldsymbol{\alpha}+\boldsymbol{\mu} t)_{i}
\end{array}\right) .
$$

Since $B$ is positive definite, $B_{i, i}>0$. By the properties of block positive definite matrix $B$, we have that

$$
\begin{aligned}
B_{I\left(t_{j}\right) I\left(t_{j}\right)}(\boldsymbol{\alpha}+\boldsymbol{\mu} t)_{I\left(t_{j}\right)}+B_{I\left(t_{j}\right), i}(\boldsymbol{\alpha}+\boldsymbol{\mu} t)_{i}= & \Sigma_{I\left(t_{j}\right), I\left(t_{j}\right)}^{-1}(\boldsymbol{\alpha}+\boldsymbol{\mu} t)_{I\left(t_{j}\right)} \\
& +B_{I\left(t_{j}\right), i}\left((\boldsymbol{\alpha}+\boldsymbol{\mu} t)_{i}-\Sigma_{i, I\left(t_{j}\right)} \Sigma_{I\left(t_{j}\right), I\left(t_{j}\right)}^{-1}(\boldsymbol{\alpha}+\boldsymbol{\mu} t)_{I\left(t_{j}\right)}\right)
\end{aligned}
$$

and

$$
B_{i, I\left(t_{j}\right)}(\boldsymbol{\alpha}+\boldsymbol{\mu} t)_{I\left(t_{j}\right)}+B_{i, i}(\boldsymbol{\alpha}+\boldsymbol{\mu} t)_{i}=B_{i, i}\left((\boldsymbol{\alpha}+\boldsymbol{\mu} t)_{i}-\Sigma_{i, I\left(t_{j}\right)} \Sigma_{I\left(t_{j}\right), I\left(t_{j}\right)}^{-1}(\boldsymbol{\alpha}+\boldsymbol{\mu} t)_{I\left(t_{j}\right)}\right)>0
$$


Then, since

$$
\Sigma_{I\left(t_{j}\right), I\left(t_{j}\right)}^{-1}\left(\boldsymbol{\alpha}+\boldsymbol{\mu} t_{j}\right)_{I\left(t_{j}\right)}>\mathbf{0}_{I\left(t_{j}\right)}, \quad\left(\boldsymbol{\alpha}+\boldsymbol{\mu} t_{j}\right)_{i}=\Sigma_{i, I\left(t_{j}\right)} \Sigma_{I\left(t_{j}\right), I\left(t_{j}\right)}^{-1}\left(\boldsymbol{\alpha}+\boldsymbol{\mu} t_{j}\right)_{I\left(t_{j}\right)}
$$

we conclude that (59) holds for all $t \in U_{j}^{o}$ such that $t-t_{j}$ is small.

On the other hand, since $I(t)=I_{j}, t \in U_{j}^{o}$ we have

$$
\begin{aligned}
& \Sigma_{I_{j} I_{j}}^{-1}(\boldsymbol{\alpha}+\boldsymbol{\mu} t)_{I_{j}}>\mathbf{0}_{I_{j}}, \\
& \Sigma_{I_{j}^{c} I_{j}} \Sigma_{I_{j} I_{j}}^{-1}(\boldsymbol{\alpha}+\boldsymbol{\mu} t)_{I_{j}}>(\boldsymbol{\alpha}+\boldsymbol{\mu} t)_{I_{j}^{c}}
\end{aligned}
$$

hold for all $t \in U_{j}^{o}$. The reason why we do not have equality in (61) is that if for some row equality holds with some $t_{1} \in U_{j}^{o}$, then $I(t)=I_{j}, t \in U_{j}^{o}$ will be invalid by linearity of the equation. Consequetly, letting $t \rightarrow t_{j}$ in the above inequalities we obtain

$$
\begin{aligned}
& \Sigma_{I_{j} I_{j}}^{-1}\left(\boldsymbol{\alpha}+\boldsymbol{\mu} t_{j}\right)_{I_{j}} \geq \mathbf{0}_{I_{j}}, \\
& \Sigma_{I_{j}^{c} I_{j}} \Sigma_{I_{j} I_{j}}^{-1}\left(\boldsymbol{\alpha}+\boldsymbol{\mu} t_{j}\right)_{I_{j}} \geq\left(\boldsymbol{\alpha}+\boldsymbol{\mu} t_{j}\right)_{I_{j}^{c}} .
\end{aligned}
$$

Suppose that the first $l$ rows (the corresponding index set is denoted by $\left.\hat{I}_{1}\right)$ of $\Sigma_{I_{j} I_{j}}^{-1}\left(\boldsymbol{\alpha}+\boldsymbol{\mu} t_{j}\right)_{I_{j}}$ are positive and the last $\sharp I_{j}-l$ rows (the corresponding index set is denoted by $\hat{I}_{2}$ ) are equal to 0 . Since $I\left(t_{j}\right)$ is the essential index set of $P_{\Sigma}\left(\boldsymbol{\alpha}+\boldsymbol{\mu} t_{j}\right)$, in view of Remark 5.1 i) we have $l \leq \sharp I\left(t_{j}\right)$. Next, as in Remark 5.1 ii) (see also the proof of Proposition 2.5 in [19]) we have

$$
\Sigma_{I_{j} I_{j}}^{-1}\left(\boldsymbol{\alpha}+\boldsymbol{\mu} t_{j}\right)_{I_{j}}=\left(\begin{array}{c}
\Sigma_{\hat{I}_{1} \hat{I}_{1}}^{-1}\left(\boldsymbol{\alpha}+\boldsymbol{\mu} t_{j}\right)_{\hat{I}_{1}} \\
\mathbf{0}_{\hat{I}_{2}}
\end{array}\right)
$$

and

$$
\Sigma_{\hat{I}_{2} \hat{I}_{1}} \Sigma_{\hat{I}_{1} \hat{I}_{1}}^{-1}\left(\boldsymbol{\alpha}+\boldsymbol{\mu} t_{j}\right)_{\hat{I}_{1}}=\left(\boldsymbol{\alpha}+\boldsymbol{\mu} t_{j}\right)_{\hat{I}_{2}}, \quad \Sigma_{\hat{I}_{1} \hat{I}_{1}}^{-1}\left(\boldsymbol{\alpha}+\boldsymbol{\mu} t_{j}\right)_{\hat{I}_{1}}>\mathbf{0}_{\hat{I}_{1}} .
$$

Then rewriting (63) we have

$$
\Sigma_{I_{j}^{c} \hat{I}_{1}} \Sigma_{\hat{I}_{1} \hat{I}_{1}}^{-1}\left(\boldsymbol{\alpha}+\boldsymbol{\mu} t_{j}\right)_{\hat{I}_{1}} \geq\left(\boldsymbol{\alpha}+\boldsymbol{\mu} t_{j}\right)_{I_{j}^{c}}
$$

which together with (64) yields that $\hat{I}_{1}$ is also an essential index set of the problem $P_{\Sigma}\left(\boldsymbol{\alpha}+\boldsymbol{\mu} t_{j}\right)$. Thus, by uniqueness, $I\left(t_{j}\right)=\hat{I}_{1} \subset I_{j}$. Consequently, $I\left(t_{j}\right) \subseteq I(t)$ for all $t \in U_{j} \cup U_{j+1}$. Finally, we show $I(t) \subseteq I\left(t_{j}\right) \cup K\left(t_{j}\right)$ for all $t \in U_{j} \cup U_{j+1}$. Since $g \in C(0, \infty)$ we have

$$
\begin{aligned}
\left(\boldsymbol{\alpha}+\boldsymbol{\mu} t_{j}\right)_{I\left(t_{j}\right)}^{\top} \Sigma_{I\left(t_{j}\right), I\left(t_{j}\right)}^{-1}\left(\boldsymbol{\alpha}+\boldsymbol{\mu} t_{j}\right)_{I\left(t_{j}\right)} & =\left(\boldsymbol{\alpha}+\boldsymbol{\mu} t_{j}\right)_{I_{j}}^{\top} \Sigma_{I_{j}, I_{j}}^{-1}\left(\boldsymbol{\alpha}+\boldsymbol{\mu} t_{j}\right)_{I_{j}} \\
& =\left(\boldsymbol{\alpha}+\boldsymbol{\mu} t_{j}\right)_{I_{j+1}}^{\top} \Sigma_{I_{j+1}, I_{j+1}}^{-1}\left(\boldsymbol{\alpha}+\boldsymbol{\mu} t_{j}\right)_{I_{j+1}} .
\end{aligned}
$$

Consequently, we conclude from Lemma 5.2 that $I_{j} \subseteq I\left(t_{j}\right) \cup K\left(t_{j}\right)$ and $I_{j+1} \subseteq I\left(t_{j}\right) \cup K\left(t_{j}\right)$, establishing the proof.

Proof of Lemma 2.2: By Lemma 5.4 for any $j=1, \ldots, q$ we have

$$
\begin{aligned}
h(t) & =\inf _{\boldsymbol{v} \geq \boldsymbol{\alpha}+\boldsymbol{\mu} t} \boldsymbol{v}^{\top} \Sigma^{-1} \boldsymbol{v} \\
& =(\boldsymbol{\alpha}+\boldsymbol{\mu} t)_{I(t)}^{\top} \Sigma_{I(t), I(t)}^{-1}(\boldsymbol{\alpha}+\boldsymbol{\mu} t)_{I(t)} \\
& =\boldsymbol{\alpha}_{I(t)}^{\top} \Sigma_{I(t), I(t)}^{-1} \boldsymbol{\alpha}_{I(t)}+2 t \boldsymbol{\alpha}_{I(t)}^{\top} \Sigma_{I(t), I(t)}^{-1} \boldsymbol{\mu}_{I(t)}+\boldsymbol{\mu}_{I(t)}^{\top} \Sigma_{I(t), I(t)}^{-1} \boldsymbol{\mu}_{I(t)} t^{2} \\
& =\boldsymbol{\alpha}_{I_{j}}^{\top} \Sigma_{I_{j}, I_{j}}^{-1} \boldsymbol{\alpha}_{I_{j}}+2 t \boldsymbol{\alpha}_{I_{j}}^{\top} \Sigma_{I_{j}, I_{j}}^{-1} \boldsymbol{\mu}_{I_{j}}+\boldsymbol{\mu}_{I_{j}}^{\top} \Sigma_{I_{j}, I_{j}}^{-1} \boldsymbol{\mu}_{I_{j}} t^{2}, \quad t \in U_{j}^{o} .
\end{aligned}
$$


Clearly, $h \in C^{1}\left(U_{j}^{o}\right)$ for all $j=1, \ldots, q$. Thus, to prove that $g \in C^{1}(0, \infty)$ it is sufficient to show that, for any $t_{j}=\overline{U_{j}} \cap \overline{U_{j+1}}$,

$$
h^{\prime}\left(t_{j}+\right)=h^{\prime}\left(t_{j}-\right)
$$

holds. It follows that

$$
\begin{aligned}
& h^{\prime}\left(t_{j}-\right)=2\left(\boldsymbol{\alpha}_{I_{j}}^{\top} \Sigma_{I_{j}, I_{j}}^{-1} \boldsymbol{\mu}_{I_{j}}+\boldsymbol{\mu}_{I_{j}}^{\top} \Sigma_{I_{j}, I_{j}}^{-1} \boldsymbol{\mu}_{I_{j}} t_{j}\right)=2 \boldsymbol{\mu}_{I_{j}}^{\top} \Sigma_{I_{j}, I_{j}}^{-1}\left(\boldsymbol{\alpha}+\boldsymbol{\mu} t_{j}\right)_{I_{j}}, \\
& h^{\prime}\left(t_{j}+\right)=2 \boldsymbol{\mu}_{I_{j+1}}^{\top} \Sigma_{I_{j+1} I_{j+1}}^{-1}\left(\boldsymbol{\alpha}+\boldsymbol{\mu} t_{j}\right)_{I_{j+1}} .
\end{aligned}
$$

Next, from Lemma 5.5 we have $I\left(t_{j}\right) \subseteq I_{j}$ and $I\left(t_{j}\right) \subseteq I_{j+1}$. For notational simplicity, we denote $B=\Sigma_{I_{j}, I_{j}}^{-1}$, $J_{j}=I_{j} \backslash I\left(t_{j}\right)$. Since

$$
\Sigma_{I_{j}, I_{j}}^{-1}(\boldsymbol{\alpha}+\boldsymbol{\mu} t)_{I_{j}}>\mathbf{0}_{I_{j}}, \quad t \in U_{j}^{o}
$$

we have

$$
\Sigma_{I_{j}, I_{j}}^{-1}\left(\boldsymbol{\alpha}+\boldsymbol{\mu} t_{j}\right)_{I_{j}} \geq \mathbf{0}_{I_{j}}
$$

Thus, by Remark 5.1 ii)

$$
\Sigma_{I_{j}, I_{j}}^{-1}\left(\boldsymbol{\alpha}+\boldsymbol{\mu} t_{j}\right)_{I_{j}}=\left(\begin{array}{c}
\Sigma_{I\left(t_{j}\right), I\left(t_{j}\right)}^{-1}\left(\boldsymbol{\alpha}+\boldsymbol{\mu} t_{j}\right)_{I\left(t_{j}\right)} \\
\mathbf{0}_{J_{j}}
\end{array}\right)
$$

with $\Sigma_{I\left(t_{j}\right), I\left(t_{j}\right)}^{-1}\left(\boldsymbol{\alpha}+\boldsymbol{\mu} t_{j}\right)_{I\left(t_{j}\right)}>\mathbf{0}_{I\left(t_{j}\right)}$ implying

$$
h^{\prime}\left(t_{j}-\right)=2 \boldsymbol{\mu}_{I_{j}}^{\top} \Sigma_{I_{j}, I_{j}}^{-1}\left(\boldsymbol{\alpha}+\boldsymbol{\mu} t_{j}\right)_{I_{j}}=2 \boldsymbol{\mu}_{I\left(t_{j}\right)}^{\top} \Sigma_{I\left(t_{j}\right), I\left(t_{j}\right)}^{-1}\left(\boldsymbol{\alpha}+\boldsymbol{\mu} t_{j}\right)_{I\left(t_{j}\right)} .
$$

Similarly, we have

$$
h^{\prime}\left(t_{j}+\right)=2 \boldsymbol{\mu}_{I\left(t_{j}\right)}^{\top} \Sigma_{I\left(t_{j}\right), I\left(t_{j}\right)}^{-1}\left(\boldsymbol{\alpha}+\boldsymbol{\mu} t_{j}\right)_{I\left(t_{j}\right)} .
$$

Consequently, $g \in C^{1}(0, \infty)$ is proved.

Now,

$$
g(t)=\frac{1}{t} \boldsymbol{\alpha}_{I_{j}}^{\top} \Sigma_{I_{j}, I_{j}}^{-1} \boldsymbol{\alpha}_{I_{j}}+2 \boldsymbol{\alpha}_{I_{j}}^{\top} \Sigma_{I_{j}, I_{j}}^{-1} \boldsymbol{\mu}_{I_{j}}+\boldsymbol{\mu}_{I_{j}}^{\top} \Sigma_{I_{j}, I_{j}}^{-1} \boldsymbol{\mu}_{I_{j}} t, \quad t \in U_{j}^{o}
$$

and

$$
g^{\prime}(t)=\frac{\boldsymbol{\mu}_{I_{j}}^{\top} \Sigma_{I_{j}, I_{j}}^{-1} \boldsymbol{\mu}_{I_{j}} t^{2}-\boldsymbol{\alpha}_{I_{j}}^{\top} \Sigma_{I_{j}, I_{j}}^{-1} \boldsymbol{\alpha}_{I_{j}}}{t^{2}}, \quad t \in U_{j}^{o} .
$$

Since for any nonempty $I_{j} \subset\{1, \ldots, d\}$

$$
\boldsymbol{\alpha}_{I_{j}}^{\top} \Sigma_{I_{j}, I_{j}}^{-1} \boldsymbol{\alpha}_{I_{j}}>0, \quad \boldsymbol{\mu}_{I_{j}}^{\top} \Sigma_{I_{j}, I_{j}}^{-1} \boldsymbol{\mu}_{I_{j}}>0
$$

we have $g(t) \rightarrow \infty$ as $t \rightarrow \infty$ and $t \rightarrow 0$, and $g^{\prime}(t)<0$ for all $t$ around $0, g^{\prime}(t)>0$ for all $t$ large enough. Thus, the function $g$ has a unique minimizer in $[0, \infty]$. Note that function $a / s+b+c s$ is decreasing to the left of some $s_{0}>0$ and increasing to the right. Consider the interval $U_{j}$. The function $g$ has a unique minimum on $U_{j}$. If at $t_{j}$ the function is decreasing it either decreasing in the whole interval, or $t_{0}$ belongs to $U_{j}$ so it is increasing at $t_{j+1}$ and consequently it is increasing at each entrance to constancy interval $U_{k}, k>j$. In this case, (12) holds and $g^{\prime}\left(t_{0}\right)=0$. Next, for $t_{0}$ we have from Lemma 5.4 that there exist some small $\varepsilon>0$ and $I^{+}, I^{-} \subseteq\{1, \ldots, d\}$ such that

$$
\begin{aligned}
& g(t)=\frac{1}{t} \boldsymbol{\alpha}_{I^{+}}^{\top} \Sigma_{I^{+} I^{+}}^{-1} \boldsymbol{\alpha}_{I^{+}}+2 \boldsymbol{\alpha}_{I^{+}}^{\top} \Sigma_{I^{+} I^{+}}^{-1} \boldsymbol{\mu}_{I^{+}}+\boldsymbol{\mu}_{I^{+}}^{\top} \Sigma_{I^{+} I^{+}}^{-1} \boldsymbol{\mu}_{I^{+}} t, \quad t \in\left(t_{0}, t_{0}+\varepsilon\right) \\
& g(t)=\frac{1}{t} \boldsymbol{\alpha}_{I^{-}}^{\top} \Sigma_{I^{-} I^{-}}^{-1} \boldsymbol{\alpha}_{I^{-}}+2 \boldsymbol{\alpha}_{I^{-}}^{\top} \Sigma_{I^{-} I^{-}}^{-1} \boldsymbol{\mu}_{I^{-}}+\boldsymbol{\mu}_{I^{-}}^{\top} \Sigma_{I^{-} I^{-}}^{-1} \boldsymbol{\mu}_{I^{-}} t, \quad t \in\left(t_{0}-\varepsilon, t_{0}\right) .
\end{aligned}
$$


Then it follows that (14) holds.

5.3. Analysis of 2-dimensional case. We now demonstrate details for Section 3.4. Recall that in our notation $I(t)$ is the essential index set of the quadradtic problem $P_{\Sigma}(\boldsymbol{\alpha}+\boldsymbol{\mu} t)$. If $I(t)^{c} \neq \emptyset$ we define

$$
K(t)=\left\{j \in I(t)^{c}: \Sigma_{j I(t)} \Sigma_{I(t) I(t)}^{-1}(\boldsymbol{\alpha}+\boldsymbol{\mu} t)_{I(t)}=(\boldsymbol{\alpha}+\boldsymbol{\mu} t)_{j}\right\}
$$

Further define

$$
b_{t}=\frac{\alpha_{2}+t}{\alpha_{1}+t} \in(0,1)
$$

It follows that

$$
g(t)=\frac{\left(\alpha_{1}+t\right)^{2}}{t} \inf _{\boldsymbol{v} \geq \boldsymbol{b}_{t}} \boldsymbol{v}^{\top} \Sigma^{-1} \boldsymbol{v}, \quad \boldsymbol{b}_{t}=\left(1, b_{t}\right)^{\top} .
$$

Case 1. $\rho<0$. Clearly $b_{t}>\rho$ and thus in view of Remark 5.1 iii) we have that $I(t)=\{1,2\}, t>0$ and

$$
\inf _{\boldsymbol{v} \geq \boldsymbol{b}_{t}} \boldsymbol{v}^{\top} \Sigma^{-1} \boldsymbol{v}=\frac{1}{1-\rho^{2}}\left(1+b_{t}^{2}-2 b_{t} \rho\right)
$$

implying

$$
g(t)=g_{1}(t):=\frac{\left(\alpha_{1}+t\right)^{2}}{t} \frac{1}{1-\rho^{2}}\left(1+b_{t}^{2}-2 b_{t} \rho\right) .
$$

Note that we slightly abuse the notation writing $g_{1}$ instead of $g_{\{1,2\}}$. It follows that for

$$
t_{0}^{(1)}=\sqrt{\frac{\alpha_{1}^{2}+\alpha_{2}^{2}-2 \alpha_{1} \alpha_{2} \rho}{2(1-\rho)}}>0
$$

we have

$$
\inf _{t \geq 0} g(t)=g_{1}\left(t_{0}^{(1)}\right)=\frac{2}{1+\rho}\left(\alpha_{1}+\alpha_{2}+2 t_{0}^{(1)}\right) .
$$

Case 2. $\rho>0$. In such a case, we have to consider if $b_{t}>\rho$ or not. Several different sub-cases are thus discussed in the following.

Case 2.1. $\alpha_{1} \rho \leq \alpha_{2}$. For this case, we have always $b_{t}>\rho, t>0$. Then $I(t)=\{1,2\}, t>0$ and $g(t)=g_{1}(t)$.

Case 2.2. $\alpha_{1} \rho>\alpha_{2}$. Let

$$
Q:=\frac{\alpha_{1} \rho-\alpha_{2}}{1-\rho}
$$

We have

(a) $\left\{b_{t}>\rho\right\} \Leftrightarrow\{t>Q\}$, for which $I(t)=\{1,2\}$,

(b) $\left\{b_{t}<\rho\right\} \Leftrightarrow\{t<Q\}$, for which $I(t)=\{1\}, K(t)=\emptyset$,

(c) $\left\{b_{t}=\rho\right\} \Leftrightarrow\{t=Q\}$, for which $I(t)=\{1\}, K(t)=\{2\}$.

Now consider (a). Since $b_{t}>\rho$, we have $g(t)=g_{1}(t), t>Q$. Now we have to check if $t_{0}^{(1)}>Q$ or not. We can show that

$$
t_{0}^{(1)}>Q \Leftrightarrow \rho<\frac{\alpha_{1}+\alpha_{2}}{2 \alpha_{1}} .
$$

Thus, we have

(a1). If $\alpha_{2} / \alpha_{1}<\rho<\frac{\alpha_{1}+\alpha_{2}}{2 \alpha_{1}}$, then $\inf _{t \in(Q, \infty)} g(t)=g_{1}\left(t_{0}^{(1)}\right)$;

(a2). If $\rho>\frac{\alpha_{1}+\alpha_{2}}{2 \alpha_{1}}$ then $\inf _{t \in(Q, \infty)} g(t)=g_{1}(Q)$. 
Next consider (b). Let $g_{2}(t)=\left(\alpha_{1}+t\right)^{2} / t$ which attains its minimum at the unique point $t_{0}^{(2)}=\alpha_{1}$. Since $b_{t}<\rho$, we have $g(t)=g_{2}(t), t \in[0, Q)$. Similarly as above we have to check if $t_{0}^{(2)}<Q$. We can show that

$$
t_{0}^{(2)}<Q \Leftrightarrow \rho>\frac{\alpha_{1}+\alpha_{2}}{2 \alpha_{1}} .
$$

Thus we have

(b1). If $\alpha_{2} / \alpha_{1}<\rho<\frac{\alpha_{1}+\alpha_{2}}{2 \alpha_{1}}$, then

$$
\inf _{t \in[0, Q)} g(t)=g_{2}(Q)=\frac{\left(\alpha_{1}-\alpha_{2}\right)^{2}}{(1-\rho)\left(\alpha_{1} \rho-\alpha_{2}\right)} .
$$

(b2). If $\rho>\frac{\alpha_{1}+\alpha_{2}}{2 \alpha_{1}}$, then

$$
\inf _{t \in[0, Q)} g(t)=g_{2}\left(t_{0}^{(2)}\right)=4 \alpha_{1} .
$$

Furthermore, by the definitions of $g_{1}, g_{2}$ and $Q$ we obtain

$$
g_{1}(Q)=g_{2}(Q)
$$

The above findings are summarized in the following lemma:

Lemma 5.6. (1). If $-1<\rho \leq \alpha_{2} / \alpha_{1}$, then $I(t)=\{1,2\}, t>0$ and

$$
t_{0}=t_{0}^{(1)}, \quad I=\{1,2\}, \quad g_{I}\left(t_{0}\right)=g_{1}\left(t_{0}^{(1)}\right), \quad g_{I}^{\prime \prime}\left(t_{0}\right)=g_{1}^{\prime \prime}\left(t_{0}^{(1)}\right)=2\left(t_{0}^{(1)}\right)^{-3} \frac{\alpha_{1}^{2}+\alpha_{2}^{2}-2 \alpha_{1} \alpha_{2} \rho}{1-\rho^{2}} .
$$

(2). If $\alpha_{2} / \alpha_{1}<\rho<\frac{\alpha_{1}+\alpha_{2}}{2 \alpha_{1}}$, then

$$
I(t)=\{1\}, \quad 0<t \leq Q, \quad I(t)=\{1,2\}, \quad t>Q
$$

and

$$
t_{0}=t_{0}^{(1)}>Q, \quad I=\{1,2\}, \quad g_{I}\left(t_{0}\right)=g_{1}\left(t_{0}^{(1)}\right), \quad g_{I}^{\prime \prime}\left(t_{0}\right)=g_{1}^{\prime \prime}\left(t_{0}^{(1)}\right) .
$$

(3). If $\rho=\frac{\alpha_{1}+\alpha_{2}}{2 \alpha_{1}}$, then

$$
I(t)=\{1\}, \quad 0<t \leq Q, \quad I(t)=\{1,2\}, \quad t>Q
$$

and

$$
t_{0}=t_{0}^{(1)}=t_{0}^{(2)}=Q, \quad I=\{1\}, \quad K=\{2\}, \quad g_{I}\left(t_{0}\right)=g_{2}\left(t_{0}^{(2)}\right), \quad g_{I}^{\prime \prime}\left(t_{0}\right)=g_{2}^{\prime \prime}\left(t_{0}^{(2)}\right)=2 \alpha_{1}^{-1}
$$

(4). If $\frac{\alpha_{1}+\alpha_{2}}{2 \alpha_{1}}<\rho<1$, then

$$
I(t)=\{1\}, \quad 0<t \leq Q, \quad I(t)=\{1,2\}, \quad t>Q
$$

and

$$
t_{0}=t_{0}^{(2)}<Q, \quad I=\{1\}, \quad K=\emptyset, \quad g_{I}\left(t_{0}\right)=g_{2}\left(t_{0}^{(2)}\right), \quad g_{I}^{\prime \prime}\left(t_{0}\right)=2 \alpha_{1}^{-1} .
$$

Remark 5.7. We point out that in general the second derivative of $g$ at $t_{0}$ is discontinuous. For instance, for the case where $\rho=\frac{\alpha_{1}+\alpha_{2}}{2 \alpha_{1}}$ in Lemma 5.6 we have

$$
g(t)=\left\{\begin{array}{cc}
\frac{1}{t}\left(\alpha_{1}+t\right)^{2}, & 0<t \leq \alpha_{1}, \\
\frac{1}{t}(\boldsymbol{\alpha}+\mathbf{1} t)^{\top} \Sigma^{-1}(\boldsymbol{\alpha}+\mathbf{1} t), & t>\alpha_{1} .
\end{array}\right.
$$


Hence

$$
g^{\prime}(t)=\left\{\begin{array}{cc}
1-\frac{\alpha_{1}^{2}}{t^{2}}, & 0<t \leq \alpha_{1}, \\
\frac{2}{1+\rho}-\frac{4 \alpha_{1}^{3}}{\left(3 \alpha_{1}+\alpha_{2}\right) t^{2}}, & t>\alpha_{1}
\end{array} \quad, \quad g^{\prime \prime}(t)=\left\{\begin{array}{cc}
\frac{2 \alpha_{1}^{2}}{t^{3}}, & 0<t \leq \alpha_{1}, \\
\frac{8 \alpha_{1}^{3}}{\left(3 \alpha_{1}+\alpha_{2}\right) t^{3}}, & t>\alpha_{1} .
\end{array}\right.\right.
$$

Consquetnly, $g \in C^{1}(0, \infty)$ is decreasing in the interval $\left(0, \alpha_{1}\right)$. Its first derivative is 0 at $t_{0}=\alpha_{1}$, however its second derivative is not continuous at $t_{0}$.

5.4. Proof of (39). Recall $R_{T}(u)$ defined in (38). We derive next sharper bounds for $P_{j ; u}\left(T, \boldsymbol{x}_{I}\right)$ and $f_{j ; u}\left(T, \boldsymbol{x}_{I}\right)$. Since $\Sigma_{J I} \Sigma_{I I}^{-1} \boldsymbol{b}_{I}<\boldsymbol{b}_{J}$, then for any small $\varepsilon>0$ and any large $Q>0$

$$
P_{j ; u}^{-}\left(T, \boldsymbol{x}_{I}, \varepsilon, Q\right) \leq P_{j ; u}\left(T, \boldsymbol{x}_{I}\right) \leq P_{j ; u}^{+}\left(T, \boldsymbol{x}_{I}, \varepsilon\right)
$$

holds for all $-N_{u} \leq j \leq N_{u}$ when $u$ is large enough, where

$$
\begin{gathered}
P_{j ; u}^{-}\left(T, \boldsymbol{x}_{I}, \varepsilon, Q\right)=\mathbb{P}\left\{\begin{array}{c}
(\boldsymbol{X}(t)-t \boldsymbol{\mu})_{I}>\boldsymbol{x}_{I} \\
\exists_{t \in[0, T]} \sqrt{t_{0}-\varepsilon} Y_{K}-\varepsilon\left|\boldsymbol{Z}_{K}\left(t, \boldsymbol{x}_{I}\right)\right|>\frac{j T}{\sqrt{u}}\left(\boldsymbol{\mu}_{K}-\Sigma_{K I} \Sigma_{I I}^{-1} \boldsymbol{\mu}_{I}\right) \\
\sqrt{t_{0}-\varepsilon} Y_{J}-\varepsilon\left|\boldsymbol{Z}_{J}\left(t, \boldsymbol{x}_{I}\right)\right|>-Q \mathbf{1}_{J}
\end{array}\right\}, \\
P_{j ; u}^{+}\left(T, \boldsymbol{x}_{I}, \varepsilon\right)=\mathbb{P}\left\{\begin{array}{c}
(\boldsymbol{X}(t)-t \boldsymbol{\mu})_{I}>\boldsymbol{x}_{I} \\
\exists_{t \in[0, T]} \sqrt{t_{0}+\varepsilon} Y_{K}+\varepsilon\left|\boldsymbol{Z}_{K}\left(t, \boldsymbol{x}_{I}\right)\right|>\frac{j T}{\sqrt{u}}\left(\boldsymbol{\mu}_{K}-\Sigma_{K I} \Sigma_{I I}^{-1} \boldsymbol{\mu}_{I}\right)
\end{array}\right\} .
\end{gathered}
$$

Furthermore, for any large $L>0$, we can find $\varepsilon>0$ sufficiently small such that

$$
e^{\frac{\boldsymbol{x}_{I}^{\top}\left(\Sigma_{I I}^{-1} \boldsymbol{b}_{I}-\boldsymbol{\varepsilon}_{I}^{\boldsymbol{x}_{I}}\right)}{t_{0}-\varepsilon\left(\boldsymbol{x}_{I}\right)}-\varepsilon} \leq f_{u}\left(T, j, \boldsymbol{x}_{I}\right)
$$

holds for all $\left\|\boldsymbol{x}_{I}\right\| \leq L$ and all $-N_{u} \leq j \leq N_{u}$ when $u$ is large enough, where

$$
\varepsilon\left(\boldsymbol{x}_{I}\right)= \begin{cases}-\varepsilon, & \boldsymbol{x}_{I}^{\top \Sigma_{I I}^{-1}} \boldsymbol{b}_{I}>0 \\ \varepsilon, & \boldsymbol{x}_{I}^{\top} \Sigma_{I I}^{-1} \boldsymbol{b}_{I} \leq 0\end{cases}
$$

and $\varepsilon_{I}^{\boldsymbol{x}_{I}}=\left(\varepsilon_{i}^{\boldsymbol{x}_{I}}, i \in I\right)$ with

$$
\varepsilon_{i}^{\boldsymbol{x}_{I}}=\left\{\begin{array}{ll}
\varepsilon, & x_{i}>0, \\
-\varepsilon, & x_{i} \leq 0,
\end{array} \quad i \in I .\right.
$$

Similarly,

$$
f_{u}\left(T, j, \boldsymbol{x}_{I}\right) \leq e^{\frac{\boldsymbol{x}_{I}^{\top}\left(\Sigma_{I I}^{-1} b_{I}+\varepsilon_{I}^{\boldsymbol{x}_{I}}\right)}{t_{0}+\varepsilon\left(\boldsymbol{x}_{I}\right)}}
$$

holds for all $\boldsymbol{x}_{I} \in \mathbb{R}^{m},-N_{u} \leq j \leq N_{u}$ when $u$ is large enough. Moreover, it follows from (16) that for the given $\varepsilon$

$$
\frac{g_{I}^{\prime \prime}\left(t_{0}\right)-\varepsilon}{2}\left(\frac{j T}{u}\right)^{2} \leq g_{I}\left(t_{0}+\frac{j T}{u}\right)-g_{I}\left(t_{0}\right) \leq \frac{g_{I}^{\prime \prime}\left(t_{0}\right)+\varepsilon}{2}\left(\frac{j T}{u}\right)^{2}
$$

holds for all $-N_{u} \leq j \leq N_{u}$ when $u$ is large enough. Consequently, we obtain the following upper bound

$$
R_{T}(u) \leq \frac{1}{\left(t_{0}-\varepsilon\right)^{m / 2}}\left(F_{1}(L, \varepsilon, T, u)+F_{2}(L, \varepsilon, T, u)\right),
$$

where

$$
\begin{aligned}
& F_{1}(L, \varepsilon, T, u)=\frac{T}{\sqrt{u}} \sum_{-N_{u}-1 \leq j \leq N_{u}} \exp \left(-\frac{g_{I}^{\prime \prime}\left(t_{0}\right)-\varepsilon}{4}\left(\frac{j T}{\sqrt{u}}\right)^{2}\right) \int_{\left\|\boldsymbol{x}_{I}\right\| \leq L} e^{\frac{\boldsymbol{x}_{I}^{\top}\left(\Sigma_{I I}^{-1} \boldsymbol{b}_{I}+\boldsymbol{\varepsilon}_{I}^{\boldsymbol{x}_{I}}\right)}{t_{0}+\varepsilon\left(\boldsymbol{x}_{I}\right)}} P_{j ; u}^{+}\left(T, \boldsymbol{x}_{I}, \varepsilon\right) d \boldsymbol{x}_{I} \\
& F_{2}(L, \varepsilon, T, u)=\frac{T}{\sqrt{u}} \sum_{-N_{u}-1 \leq j \leq N_{u}} \exp \left(-\frac{g_{I}^{\prime \prime}\left(t_{0}\right)-\varepsilon}{4}\left(\frac{j T}{\sqrt{u}}\right)^{2}\right)
\end{aligned}
$$




$$
\times \int_{\mid \boldsymbol{x}_{I} \|>L} e^{e_{\frac{\boldsymbol{x}_{I}^{\top}\left(\Sigma_{I I}^{-1} b_{I}+\boldsymbol{e}_{I}^{\boldsymbol{x}_{I}}\right)}{t_{0}+\varepsilon\left(\boldsymbol{x}_{I}\right)}}} \mathbb{P}\left\{\exists_{t \in[0, T]}(\boldsymbol{X}(t)-t \boldsymbol{\mu})_{I}>\boldsymbol{x}_{I}\right\} d \boldsymbol{x}_{I}
$$

Next, it follows that

$$
\lim _{\varepsilon \rightarrow 0} \lim _{u \rightarrow 0} F_{1}(L, \varepsilon, T, u)=\int_{\left\|\boldsymbol{x}_{I}\right\| \leq L} e^{\frac{\boldsymbol{x}_{I}^{\top} \Sigma_{I I}^{-1} \boldsymbol{b}_{I}}{t_{0}}} \mathbb{P}\left\{\exists_{t \in[0, T]}(\boldsymbol{X}(t)-t \boldsymbol{\mu})_{I}>\boldsymbol{x}_{I}\right\} d \boldsymbol{x}_{I} \int_{-\infty}^{\infty} e^{-\frac{g_{I}^{\prime \prime}\left(t_{0}\right) x^{2}}{4}} \psi(x) d x
$$

and

$$
\lim _{\varepsilon \rightarrow 0} \lim _{u \rightarrow 0} \frac{T}{\sqrt{u}} \sum_{-N_{u}-1 \leq j \leq N_{u}} \exp \left(-\frac{g_{I}^{\prime \prime}\left(t_{0}\right)-\varepsilon}{4}\left(\frac{j T}{\sqrt{u}}\right)^{2}\right)=\int_{-\infty}^{\infty} e^{-\frac{g_{I}^{\prime \prime}\left(t_{0}\right) x^{2}}{4}} d x .
$$

Hence in view of Lemma 4.2, letting $L \rightarrow \infty$ we obtain

$$
\lim _{u \rightarrow \infty} R_{T}(u) \leq \frac{1}{t_{0}^{m / 2}} \mathcal{H}_{I}(T) \int_{-\infty}^{\infty} e^{-\frac{g_{I}^{\prime \prime}\left(t_{0}\right) y^{2}}{4}} \psi(y) d y .
$$

Similarly, we obtain the following lower bound

$$
R_{T}(u) \geq \frac{1}{\left(t_{0}+\varepsilon\right)^{m / 2}} F_{3}(L, Q, \varepsilon, T, u),
$$

where

$$
\begin{aligned}
F_{3}(L, Q, \varepsilon, T, u)= & \frac{T}{\sqrt{u}} \sum_{-N_{u}-1 \leq j \leq N_{u}} \exp \left(-\frac{g_{I}^{\prime \prime}\left(t_{0}\right)+\varepsilon}{4}\left(\frac{j T}{\sqrt{u}}\right)^{2}\right) \\
& \times \int_{\left\|\boldsymbol{x}_{I}\right\| \leq L} e^{\frac{\boldsymbol{x}_{I}^{\top}\left(\Sigma_{I I}^{-1} \boldsymbol{b}_{I}-\boldsymbol{\varepsilon}_{I}^{\boldsymbol{x}_{I}}\right)}{t_{0}-\varepsilon\left(\boldsymbol{x}_{I}\right)}-\varepsilon} P_{j ; u}^{-}\left(T, \boldsymbol{x}_{I}, \varepsilon, Q\right) d \boldsymbol{x}_{I} .
\end{aligned}
$$

Letting $u \rightarrow \infty, Q \rightarrow \infty, \varepsilon \rightarrow 0, L \rightarrow \infty$ (in this order) and in view of Lemma 4.2 we obtain

$$
\lim _{u \rightarrow \infty} R_{T}(u) \geq \frac{1}{t_{0}^{m / 2}} \mathcal{H}_{I}(T) \int_{-\infty}^{\infty} e^{-\frac{g_{I}^{\prime \prime}\left(t_{0}\right) y^{2}}{4}} \psi(y) d y>0 .
$$

Consequently, the claim follows and the proof is complete.

Acknowledgement: Thanks to Swiss National Science Foundation grant No.200021-166274. TR \& KD acknowledge partial support by NCN Grant No 2015/17/B/ST1/01102 (2016-2019).

\section{REFERENCES}

[1] C. Knessl and J. B. Keller, "Probability of Brownian motion hitting an obstacle," SIAM Journal on Applied Mathematics, vol. 60, no. 2, pp. 729-745, 2000.

[2] H. J. Landau, B. F. Logan, L. A. Shepp, and N. Bauman, "Diffusion, cell mobility, and bandlimited functions," SIAM Journal on Applied Mathematics, vol. 44, no. 6, pp. 1232-1245, 1984.

[3] J. J. Bowman, T. B. A. Senior, and P. L. E. Uslenghi, Electromagnetic and acoustic scattering by simple shapes (Revised edition). New York, Hemisphere Publishing Corp., 1987.

[4] R. Garbit and K. Raschel, "On the exit time from a cone for Brownian motion with drift," Electron. J. Probab., vol. 19, pp. 1-27, 2014.

[5] Z. Puchała and T. Rolski, "The exact asymptotic of the collision time tail distribution for independent Brownian particles with different drifts," Probability Theory and Related Fields, vol. 142, no. 3, pp. 595-617, 2008.

[6] J. Hüsler and V. I. Piterbarg, "A limit theorem for the time of ruin in a Gaussian ruin problem," Stochastic Process. Appl., vol. 118, no. 11, pp. 2014-2021, 2008.

[7] K. Dẹbicki, E. Hashorva, and L. Ji, "Parisian ruin of self-similar Gaussian risk processes," J. Appl. Probab, vol. 52, pp. 688-702, 2015. 
[8] K. Dębicki, E. Hashorva, and L. Ji, "Gaussian risk models with financial constraints," Scand. Actuar. J., no. 6, pp. 469-481, 2015.

[9] K. Dẹbicki, K. M. Kosiński, M. Mandjes, and T. Rolski, "Extremes of multidimensional Gaussian processes," Stochastic Process. Appl., vol. 120, no. 12, pp. 2289-2301, 2010.

[10] J. Pickands, III, "Upcrossing probabilities for stationary Gaussian processes," Trans. Amer. Math. Soc., vol. 145, pp. 51-73, 1969.

[11] J. Pickands, III, "Maxima of stationary Gaussian processes," Z. Wahrscheinlichkeitstheorie und Verw. Gebiete, vol. 7, pp. 190-223, 1967.

[12] V. I. Piterbarg, Asymptotic methods in the theory of Gaussian processes and fields, vol. 148 of Translations of Mathematical Monographs. Providence, RI: American Mathematical Society, 1996.

[13] K. Dębicki, Z. Michna, and T. Rolski, "Simulation of the asymptotic constant in some fluid models," Stoch. Models, vol. 19, no. 3, pp. 407-423, 2003.

[14] A. J. Harper, "Bounds on the suprema of Gaussian processes, and omega results for the sum of a random multiplicative function," Ann. Appl. Probab., vol. 23, no. 2, pp. 584-616, 2013.

[15] A. B. Dieker and T. Mikosch, "Exact simulation of Brown-Resnick random fields at a finite number of locations," Extremes, vol. 18, pp. 301-314, 2015.

[16] D. Cheng, "Excursion probabilities of isotropic and locally isotropic Gaussian random fields on manifolds," Extremes, in press.

[17] K. Dȩbicki, S. Engelke, and E. Hashorva, "Generalized Pickands constants and stationary max-stable processes," arXiv:1602.01613, 2016.

[18] E. Hashorva, "Asymptotics and bounds for multivariate Gaussian tails," J. Theoret. Probab., vol. 18, no. 1, pp. 79-97, 2005.

[19] E. Hashorva and J. Hüsler, "On asymptotics of multivariate integrals with applications to records," Stochastic Models, vol. 18, no. 1, pp. 41-69, 2002.

[20] K. Dȩbicki, E. Hashorva, L. Ji, and K. Tabiś, "Extremes of vector-valued Gaussian processes: Exact asymptotics," Stochastic Process. Appl., vol. 125, pp. 4039-4065, 2015.

[21] K. Joag-Dev and F. Proshan, "Negative association of random variables with applications," The Annals of Statistics, vol. 11, pp. 286-295, 1983.

[22] G. Samorodnitsky, "Probability tails of Gaussian extrema," Stochastic Process. Appl., vol. 38, no. 1, pp. 55-84, 1991.

[23] R. Adler and J. Taylor, Random fields and geometry. Springer Monographs in Mathematics, New York: Springer, 2007.

[24] J. Azaïs and M. Wschebor, Level sets and extrema of random processes and fields. Hoboken, NJ: John Wiley \& Sons Inc., 2009.

[25] M. Lifshits, Lectures on Gaussian processes. Springer Briefs in Mathematics, Springer, Heidelberg, 2012.

[26] K. Dȩbicki, E. Hashorva, and P. Liu, "Ruin probabilities and passage times of $\gamma$-reflected Gaussian process with stationary increments," http://arXiv.org/abs/1511.09234, 2015.

[27] V. I. Piterbarg, "High extrema of Gaussian chaos processes," Extremes, vol. 19, no. 2, pp. 253-272, 2016. 
Krzysztof Dȩbicki, Mathematical Institute, University of WrocŁaw, Pl. Grunwaldzki 2/4, 50-384 Wroceaw, Poland

E-mail address: Krzysztof.Debicki@math.uni.wroc.pl

Enkelejd Hashorva, Department of Actuarial Science, University of Lausanne, UNiL-Dorigny, 1015 Lausanne, SwitzerLAND

E-mail address: Enkelejd.Hashorva@unil.ch

Lanpeng Ji, Department of Actuarial Science, University of Lausanne, UniL-Dorigny, 1015 Lausanne, Switzerland

E-mail address: jilanpeng@126.com

Tomasz Rolski, Mathematical Institute, University of WrocŁaW, Pl. Grunwaldzki 2/4, 50-384 WrocŁaW, Poland

E-mail address: Tomasz.Rolski@math.uni.wroc.pl 